\section{BOLLETINO DELLA \\ SOCIETA' CEOCRAFICA ITALIANA}

OPEN ACCESS

Citation: E. Guadagno, E. Manzi (2021). La prego di voler gradire una mia Carta dei prodotti alimentari.... Bollettino della Società Geografica Italiana serie 14, 4(1): 19-37. doi: 10.36253/bsgi-1249

Copyright: (c) 2021 E. Guadagno, E. Manzi. This is an open access, peerreviewed article published by Firenze University Press (http://www.fupress. com/bsgi) and distributed under the terms of the Creative Commons Attribution License, which permits unrestricted use, distribution, and reproduction in any medium, provided the original author and source are credited.

Data Availability Statement: All relevant data are within the paper and its Supporting Information files.

Competing Interests: The Author(s) declare(s) no conflict of interest.

For Italian Evaluation Purposes: Elio Manzi is Author of sections 1, 5, 6; Eleonora Guadagno is Author of sections 2, 3, 4.
Firenze University Press

www.fupress.com/bsgi

\title{
La prego di voler gradire una mia Carta dei prodotti alimentari...
}

\section{Dear Mr. Fiorelli, I would like you to accept as a gift my Map of Nourishing Products of the Kingdom of Two Sicilies...}

\author{
Eleonora Guadagno ${ }^{1}$, Elio Manzi ${ }^{2}$ \\ ${ }^{1}$ Dipartimento di Scienze Umane e Sociali, Università degli Studi di Napoli "L'Orientale", \\ Italia \\ ${ }^{2}$ Già Università degli Studi di Palermo, Italia \\ E-mail: eguadagno@unior.it; elio.manzi.mw4t@alice.it
}

\begin{abstract}
The Authors examine some elements of the work of the great cartographer Benedetto Marzolla (1801-1858) who, together with Carlo Afàn de Rivera, represents the best among the intellectuals and technicians that the Kingdom of Two Sicilies yielded before the Italian unification. This contrasts with the commonplace outlined be the Savoyard-Risorgimental propaganda which describes Naples and the Kingdom as a country inhabited by incapables, depraved and lazy persons. The Authors resume the theme of the Map of Nourishing Products, an excellent work not only because it describes the terrestrial and marine nourishing productions, but also for the connection with many agrarian landscapes and relative trades. Moreover, the Authors present the Map of Excise Duty (1830) another work of Marzolla together with Valentino, never known or considered by cartography scholars. This map does not seem to be cited inside the catalogues or inventories about Marzolla's production, compiled by Valerio or by others authors in the frame of the analysis of the Royal Topographical Office of Naples (ROT), especially along the second half of the XIX century. The "modernity" of Marzolla manifests not only in consideration of anti-historical comparisons with actual excellent or "organic" food products, but for the analogies with the original proposals of Brunet, Ferras and other academics of the Maison de la Géographie. Unfortunately, Italian geographers have devoted little attention to this field of study.
\end{abstract}

Keywords: Benedetto Marzolla, History of cartography, Kingdom of Two Sicilies, nourishing typical products.

Riassunto. Disamina sul grande cartografo Benedetto Marzolla (1801-1858) il quale, assieme a Carlo Afàn de Rivera, rappresentò quanto di meglio a livello intellettuale e tecnico il Regno delle Due Sicilie produsse nell'ultimo periodo di vita, prima dell'Unità d'Italia. Questi importanti e concreti intellettuali smentiscono una parte della propaganda sabaudo-risorgimentale su Napoli e il Mezzogiorno, abitato, secondo tali luoghi comuni offensivi, solo da ignoranti, incapaci, corrotti e infingardi. Si riprende il tema della Carta dei Prodotti alimentari, opera significativa per la ricostruzione non solo delle produzioni alimentari sia di terra che di mare, ma anche dei paesaggi agrari e dei commerci. Viene presentata la Carta dei Dazi del Regno (1830) altro lavoro precedente di Marzolla e di Valentino, sinora mai commentata o resa nota agli studiosi. La carta 
non è citata nei pur accurati elenchi della produzione di Marzolla, redatti nell'ambito degli studi sul Reale Officio Topografico di Napoli (ROT), specie nell'Ottocento o all'inizio del Novecento. La "modernità" di Marzolla si evidenzia non tanto nei paragoni spesso antistorici con i prodotti attuali di eccellenza, quanto nelle analogie con le originali concezioni cartografiche di Brunet, Ferras e altri della Maison de la Géographie. Purtroppo la geografia italiana ha poco praticato questi filoni di studio.

Parole chiave: Benedetto Marzolla, storia della cartografia, Regno delle Due Sicilie, prodotti tipici alimentari.

\section{Introduzione}

Così Benedetto Marzolla scriveva il 27 gennaio 1857 a Giuseppe Fiorelli, il futuro famoso archeologo di Pompei. Il grande cartografo brindisino con la modestia e cortesia che gli erano congeniali, ricordava al Fiorelli, allora segretario del Conte di Siracusa, fratello del Re, di avergli inviato una copia del suo originale prodotto cartografico, una delle più gustose realizzazioni tematiche dell'Ottocento (Biblioteca Nazionale di Napoli [BNN], Ms. S.Mart.780.75).

Marzolla sarebbe scomparso poco dopo, nel 1858, colto da infarto, lasciando incompiute alcune opere cui attendeva. Così ci fa sapere il fratello Raffaele, alto magistrato, in un accurato necrologio che resta la migliore biografia sul valente cartografo (Marzolla R. 1858). Come per altri grandi, siano tecnici o scienziati, artisti figurativi, scrittori, ci chiediamo quali nuove importanti realizzazioni ci avrebbe potuto lasciare senza la fine prematura. Un rimpianto un po' ingenuo ma spontaneo.

Tuttavia la fine di Marzolla è quasi parallela a quella di Carlo Afàn de Rivera, un altro personaggio emblematico dell'alta qualità degli studi e delle opere a stampa fiorite nel Regno delle Due Sicilie e segnatamente a Napoli, luci vivide in un ambiente e un'epoca abitati anche da mediocri esecutori o da tecnici capaci, ma non geniali come i due appena ricordati. Ma non certo un ambiente esclusivamente popolato da incapaci, corrotti, ignoranti e infingardi, come una parte della propaganda risorgimentale e sabauda volle presentare.

La morte del direttore di Ponti e Strade e del proprietario e animatore del più originale laboratorio cartografico napoletano, appena prima della bufera unitaria seguita alla spedizione dei Mille, è simbolica della fine di un mondo criticabile ma indipendente, dove personalità scientifiche e operatori pratici nulla avevano da invidiare, in qualche caso, alle migliori espressioni europee. Lo stesso personaggio ricordato pocanzi, cui Marzolla indirizzava la lettera, fu l'ideatore del metodo con il quale ancor oggi si ricavano i calchi dei morti - umani o animali - che l'eruzione vesuviana del 79 d.C. aveva disseminato nella famosa città sepolta, colando del gesso liquido nella cavità lasciata attorno al corpo, semi-dissolto dai lapilli e dalla cenere roventi. Fiorelli fu nominato direttore di Pompei poco dopo l'Unità, per l'abilità professionale ma anche per meriti di "salto della quaglia”, per così dire, nel senso di aver fatto la fronda, seppur tardiva, verso il regime borbonico. Non sappiamo come si sarebbero comportati Marzolla e Afàn De Rivera se fossero ancora vissuti. Ma forse il destino risparmiò loro umiliazioni pratiche o morali.

L'attenzione recente di numerosi studiosi si è appuntata su due prodotti cartografici molto diversi tra loro e di epoche differenti: le cosiddette "carte aragonesi" e la Carta dei Prodotti alimentari di Benedetto Marzolla. Un'attenzione quasi mediatica, seppur ristretta al limitato settore degli specialisti, per entrambe. Più accentuata per la Carta dei Prodotti per motivi pratici, trattandosi di un'unica opera in scala al milionesimo, di semplice ed immediata lettura e facilmente usabile con forzature per paralleli e strumentalizzazioni di attualità, specie localistiche. Infatti nel sito web di alcune regioni e di qualche comune, la carta marzolliana viene citata. La vicenda della cartografia aragonese del Regno di Napoli è più complessa e quindi necessita di competenze abbastanza elevate, per cui se ne sono occupati quasi solo storici di professione o specialisti di alta caratura come Vladimiro Valerio $o$, in qualche caso, archivisti, cioè storici del documento: un improvviso risveglio dopo un lungo oblio nonostante che la strada fosse stata tracciata a cavallo tra Ottocento e Novecento da Aldo Blessich. Ma non è questa la sede per riprendere il tema "carte aragonesi", vicenda che chi scrive ha recentemente sintetizzato su L'Universo, la rivista dell'Istituto Geografico Militare (Manzi 2018).

La curiosa ma interessante Carta dei Prodotti, scrivevamo nel lontano 1975 in occasione della Mostra cartografica del XXII Congresso Geografico Italiano da lui curata, è una

“gustosa 'curiosità' cartografica, un po' esulante dal tema della mostra, tuttavia meritevole di interesse. La tavola geo-pittorica illustra le principali, tipiche produzioni agricole delle varie regioni meridionali, dalle più celebri (come per la Campania Felice) a quelle meno note (come per la Basilicata). Produzioni perdurate sino a pochi anni addietro, sino, cioè, alla sistematica, recente distruzione dell'agricoltura meridionale" (Manzi 1977, 198).

Nel 1987, quando chi scrive, convinto dall'amico e collega Mario Cataudella, uno dei massimi esperti di cartografia in Italia, a collaborare ad un Atlante Regionale della Basilicata da lui diretto, su incarico di quella 
Giunta Regionale, accettò di curare la sezione di geografia storica nella quale, Tav. III, fu inserita la Carta dei Prodotti, con un "esploso" ingrandito sulla Basilicata e un commento, che in parte più avanti viene ripreso in questo articolo. Dopo questi due contributi "pionieri”, per così dire, più di recente il tema è stato trattato con una completa ricostruzione bibliografica da Silvia Siniscalchi dell'Università di Salerno (Siniscalchi 2019), mentre la precedente disamina di Simonetta Conti del 2008, comparsa nel volume in onore di Carmelo Formica, reca una bibliografia sintetica.

Più o meno in contemporanea con Manzi, la Carta dei Prodotti venne ricordata in uno scritto poco noto di Domenico Novembre, geografo salentino del secolo scorso (Novembre 1972). Ora, dev'essere chiaro che nessuno ha effettuato mirabolanti "scoperte" col porre attenzione su quest'opera del grande cartografo, la quale, in più copie, dalla sua morte in poi è rimasta nei fondi che via via, dalla Reale Biblioteca Borbonica e il suo Fondo Palatino e altri, hanno formato l'attuale Biblioteca Nazionale di Napoli. La carta si trova anche in altre istituzioni storiche e bibliotecarie di Napoli e altrove. Le "scoperte" di documenti esistenti nelle biblioteche o cartoteche, schedati, le lasciamo a geniali scopritori dell'acqua calda.

Comunque, antesignano nella citazione della Carta dei Prodotti fu Angelo De Fabrizio nel 1925, in un articolo pubblicato sull'Annuario 1923-1924 del R. Ginnasio "Benedetto Marzolla" di Brindisi, lo stesso Istituto che molti anni dopo ha promosso, assieme all'amministrazione comunale brindisina, la mostra in onore del cartografo, da cui Valerio ha tratto il bel volume del 2008. Invece Gustavo Mazzarelli, altro studioso del Marzolla tra le due guerre, in un articolo del 1930 tratta solo degli atlanti, non della carta tematica alimentare.

De Fabrizio e Mazzarelli pongono l'accento su aspetti di modernità, buona informazione e spunti "nazionalistici" ante litteram, ad esempio sull'Istria italiana, secondo il costume del tempo, specie Mazzarelli, che scrive in pieno fascismo. Il nazionalismo proclamato sia durante il fascismo sia, rivisitato come "sovranismo" ai nostri giorni, conduce sempre a risultati opposti a quelli in teoria auspicati, come fu dimostrato dalla perdita dei territori orientali italiani acquisiti con la prima guerra mondiale, dopo la disastrosa partecipazione alla seconda guerra.

Di recente, invece, Marzolla, e in particolare la Carta dei Prodotti, viene adoperato per considerazioni in parte antistoriche, forzando e attualizzando il significato dell'opera, che è importante piuttosto come documento sulla relativamente florida economia agricola e d'allevamento in epoca preunitaria, quando la fame profonda e la tragedia dell'emigrazione dal Mezzogiorno erano ancora sconosciute. Una tragedia svoltasi tra gli ultimi decenni dell'Ottocento e l'inizio del Novecento, cui non fu estranea la politica di ignoranza ambientale dei governi sabaudi, con la distruzione di vaste aree forestali (in Basilicata e Calabria) e di acquisizione delle terre da parte di voraci ex-dipendenti delle casate aristocratiche, e la fine del diritto di legnatico, spigolatura e piccola coltivazione temporanea che dava sollievo alle frange più povere delle popolazioni. L'attualizzazione forzata ricorda un po' le regie teatrali "d'avanguardia" laddove le opere liriche sono ambientate a forza ai nostri giorni, secoli dopo il periodo immaginato dagli autori.

La carta attira l'occhio perché di lettura immediata, di semplice costruzione, stampata in nitida litografia, arte in cui Marzolla era maestro, e non reca dati statistici che appesantirebbero l'immediatezza visiva, quei dati che hanno reso la geografia scolasticamente odiosa in un paese a-geografico come l'Italia. Invece la storia di Napoli e del Mezzogiorno è lunga e complessa, non racchiudibile in nozioni scolastiche di impronta risorgimentale. Come nemmeno nelle iperboli neoborboniche, posizioni che riteniamo entrambe dannose e anticulturali.

La storia d'Italia non comincia nel 1861 (17 marzo, proclamazione del Regno d'Italia) perché esistevano stati, istituzioni, operatori tecnici e culturali di valore anche prima, e tuttavia la propaganda scolastica sia nei primi decenni post-unitari sia dopo la caduta del fascismo, condusse a convinzioni di fatto anti-meridionali, con lo sminuire l'eccellenza della cartografia napoletana e meridionale.

Tre esempi. Vladimiro Valerio, ottimo studioso di storia della cartografia del Mezzogiorno (e non solo), autore di opere esaustive e di vasta portata interdisciplinare, a giudizio di chi scrive talora tende a esagerare le condanne della dinastia borbonica che, pur tra remore ed errori, ebbe il merito di avviare la grande stagione della cartografia napoletana settecentesca e poi di tollerare in posti di rilievo personaggi di valore scientifico e tecnico, se non apertamente e ripetutamente compromessi. Forse questa tendenza di Valerio si deve alla sua ammirazione per Ferdinando Visconti, studioso e cartografo di livello internazionale, prosecutore di Rizzi Zannoni nel progetto a lungo termine della copertura cartografica del Regno.

Attilio Mori, direttore della biblioteca e cartoteca dell'Istituto Geografico Militare tra le due guerre, pubblica nel cinquantenario dell'Istituto un'ampia rassegna sulla cartografia ufficiale, nella quale dà notevole spazio alle vicende di Napoli pre-unitaria (e come avrebbe potuto non farlo?) ma senza evidenziare la superiorità tecnica e artistica. Eravamo in pieno periodo sabaudo.

Aldo Blessich, apripista fondamentale sia per le "carte aragonesi" sia per ogni ulteriore analisi sulla storia della 
cartografia napoletana, rimase quasi ignorato negli studi successivi, o solo a fatica ricordato nella bibliografia.

Marzolla ha sempre esercitato un certo fascino sugli studiosi, per la bellezza delle esecuzioni, per la chiarezza di lettura, per la funzione di "supplenza" della cartografia ufficiale del Mezzogiorno, dovuta a vicende politiche soprattutto. Una limitata valorizzazione di due personaggi dalle notevoli capacità tecniche e comunicative, come Carlo Afàn de Rivera e appunto Benedetto Marzolla, è stata effettuata di recente. Entrambi muoiono prima dell'Unità, e, come tanto altro del patrimonio culturale del Mezzogiorno, obliati, trascurati, sminuiti per motivi di propaganda e per il principio antico di "guai ai vinti". Infatti, il Regno delle Due Sicilie fu l'unica entità statale che fece la guerra prima e la guerriglia poi al nascente stato italiano unitario. Le radici storiche dell'anti-meridionalismo, mai morte, si risvegliano prepotentemente a partire dagli anni Novanta del Novecento. Non è questa la sede per spendere le lodi di Carlo Afàn de Rivera, per il quale si rimanda a vecchi e recenti studi (Manzi 1977; 2007b) e pure a un piccolo prezioso articolo di Lucio Gambi (1954) e a lavori di Di Blasio (1993; 2004). Marzolla, invece, conosce di recente una "popolarità" nuova, certo meritata, alla quale vogliamo aggiungere questo modesto contributo di approfondimento.

\section{Il "valore" della Carta dei Prodotti e i suoi dettagli}

Il valore innovativo della Carta dei Prodotti Alimentari viene riconosciuto dallo stesso Marzolla il quale, come accennato nell'introduzione, la rende oggetto di omaggio a Giuseppe Fiorelli. Nel periodo in cui l'archeologo faceva parte a pieno titolo dell'entourage del Conte di Siracusa, mecenate di molti ritrovamenti in Campania, Marzolla gli si rivolge per fare da tramite nel "raccomandare" proprio al fratello del Re un "giovane di ingegno non comune", Concetto Caravella. In calce alla lettera, datata 27 gennaio 1857, si legge "La prego di voler gradire una copia della carta dei prodotti alimentari da me eseguita per incarico del Ministro dell'interno a cui fece legarne copia per uso di S.A.R" [sottolineatura originale].

Questa lettera testimonia il valore della produzione di Marzolla anche tra i coevi e, in effetti, Marzolla inviava in regalo a vari personaggi i propri lavori cartografici, ovviamente al sovrano e a esponenti politici di spicco, ma anche a varie altre persone interessate per motivi scientifici, artistici, amministrativi o semplicemente come ricordo visivo, certamente più efficace di un semplice scritto. La valenza simbolica della carta che mostra

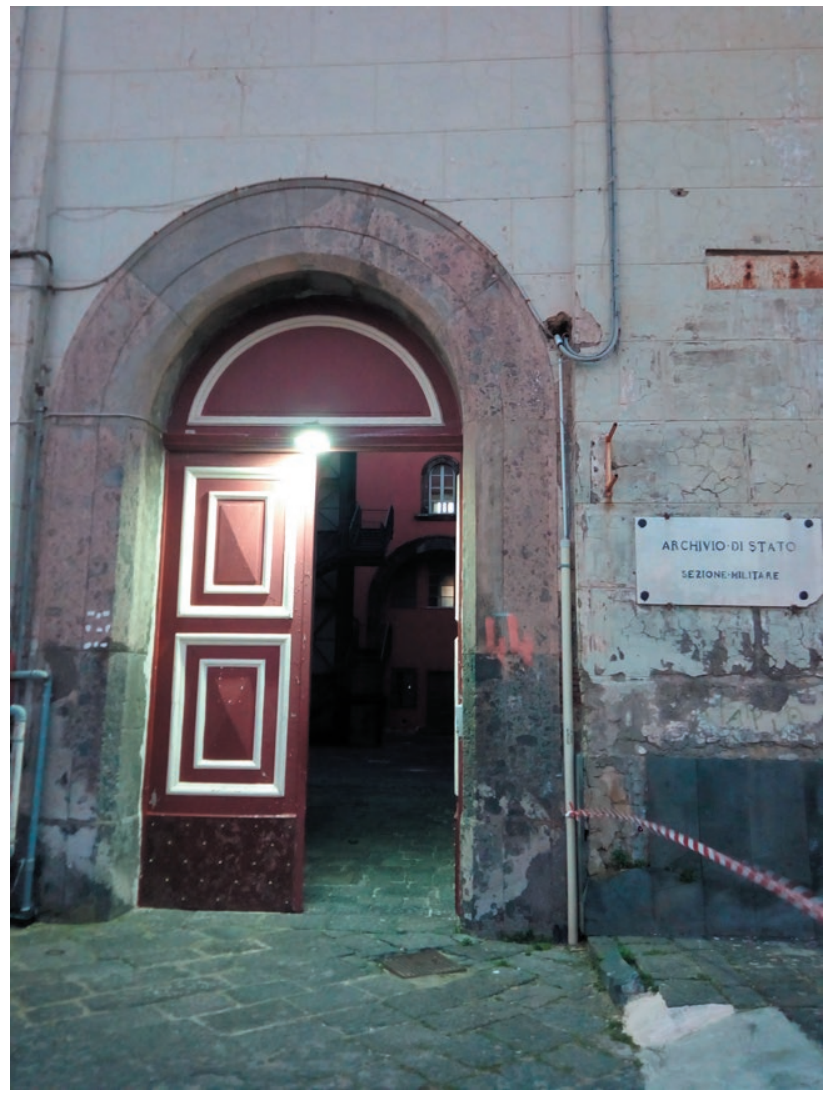

Figura 1. Napoli. Uno scorcio del palazzetto dell'Archivio di Stato, Sezione Militare a Pizzofalcone, ingresso all'Archivio. Fu sede del Reale Officio Topografico di Napoli (ROT), dove Marzolla lavorò per lunghi anni (Foto E. Guadagno).

le specificità della produzione agroalimentare del Mezzogiorno nel 1856, esclusa la Sicilia (a parte l'isola di Lipari), può essere osservata da diverse angolature, già messe in evidenza in più occasioni (Manzi 2007a).

La carta, secondo quanto riportato da Siniscalchi $(2019,70)$, inoltre, è "vivacizzata" da un cartiglio i cui dettagli sono già riportati in Manzi (2007a). Il minuto cartiglio è frutto di un'antica tradizione di decorazione cartografica allegorica, in cui vengono inserite le informazioni che identificano il documento: titolo, autore, incisore, luogo, data di edizione, scala e didascalie. Proprio come il manufatto cartografico, anche il suo aspetto estetico è molto presente nei tempi moderni: è il "luogo" dove l'incisore esprime il suo talento ${ }^{1}$.

\footnotetext{
${ }^{1}$ Tra i primi studi sui cartigli (cartouches in francese e in inglese, cartuchos in spagnolo), già presenti nelle figurazioni egizie su papiro e talora in affreschi e bassorilievi, si ricordano quelli messi a punto da François de Dainville il quale fa fare alla storia della cartografia un "salto epistemologico" mentre sposta l'attenzione su nuovi oggetti di studio, tutti analizzati nel suo Cartes anciennes de l'Eglise de France,
} 
Il cartiglio della Carta dei Prodotti alimentari raffigura un banchetto al quale stanno partecipando quattro persone, due donne e due uomini: di questi ultimi, uno è in piedi mentre degusta un calice di vino, un altro è seduto con una bottiglia di vino in mano. Il vino che sorseggiano i commensali, forse dal colore paglierino, potrebbe dirsi Asprinio $^{2}$, una varietà a bacca bianca, tipico dell'area, selezionato originariamente da Louis Pierrefeu, il cantiniere della corte di Roberto d'Angiò ${ }^{3}$.

La scena - all'aperto - si svolge su una delle fertili colline dell'area flegrea (ad occidente di Napoli) e il banchetto è allestito rusticamente su un tavolo di legno intorno al quale ci sono sedie in legno stile "Vienna Thonet", tipiche del tempo: alle spalle, nell'entroterra, un'area urbana, dal quale svetta una cupola. Il terreno vulcanico che calpestano sembra costellato di lapilli. Fanno poi, da cornice, sullo sfondo, il Vesuvio fumante (secondo il catalogo storico dell'INGV, l'ultima eruzione effusiva c'era stata nel 1855 e il Re aveva visitato il sito e l'osservatorio) e una vite sulla destra, con abbondanti grappoli nonché, in basso, due cornucopie - simbolo d'abbondanza - stracolme di frutta, ortaggi e grano, che si intrecciano.

Ad ogni modo, il recupero delle tradizioni locali associate alla "riscoperta" di prodotti tipici (si pensi al recente Atlante dei prodotti tipici locali dei Parchi Italiani) quali segni distintivi del made in Italy legati alla preoccupazione tutta post-moderna della ricerca del cibo genuino e della Lebenfürsorge, termine tedesco indicante la "cura della vita" che pare addirittura aver soppiantato quello anglofono di wellness, non deve essere confuso con la ricostruzione della tipicità offerta dalla Carta. I marchi di qualità sono dei brevetti che assicurano un modo di contraddistinguere il prodotto o

come, appunto, la storia delle tecniche cartografiche (incisione, miniatura, stampa), la distribuzione dei documenti e il loro status, gli attori politici e sociali nella cartografia, i "margini" dei documenti (ornamenti e, dunque, cartigli) (Palsky 2018, 89).

${ }^{2}$ Il vino Asprinio di Aversa ha ottenuto il riconoscimento della Denominazione di Origine Controllata con Decreto Presidente della Repubblica del 31/07/1993, modificato con successivo Decreto Ministeriale del 09/02/1994.

${ }^{3}$ I vitigni che crescono su terreno tufaceo, noto proprio come tufo giallo, originato dal distretto vulcanico dei Campi Flegrei e connessi all'eruzione della cosiddetta Ignimbrite Campana e piantati a ridosso di alberi di pioppo (più raramente olmo) garantiscono una produzione "in altezza" (anche 15-20 m) di tale varietà; le viti, per questo motivo vengono chiamate "maritate" e il paesaggio che ne deriva "alberata aversana" (Assessorato all'Agricoltura, Regione Campania, 2006). Le prime attestazioni "letterarie" del vino si ritrovano in Lancerio (1500-1550), bottigliere del Papa Paolo III Farnese, "Il vino asprino viene da un luogo vicino a Napoli. Li migliori sono quelli di Aversa, città unica e buona. Bianchi e rossi, questi sono meglio. Tali vini sono molto crudi, sono vini da podagrosi. L'estate è sana bevanda" (secondo quanto riportato da Ferraro nel 1879, in Celentano 2004)

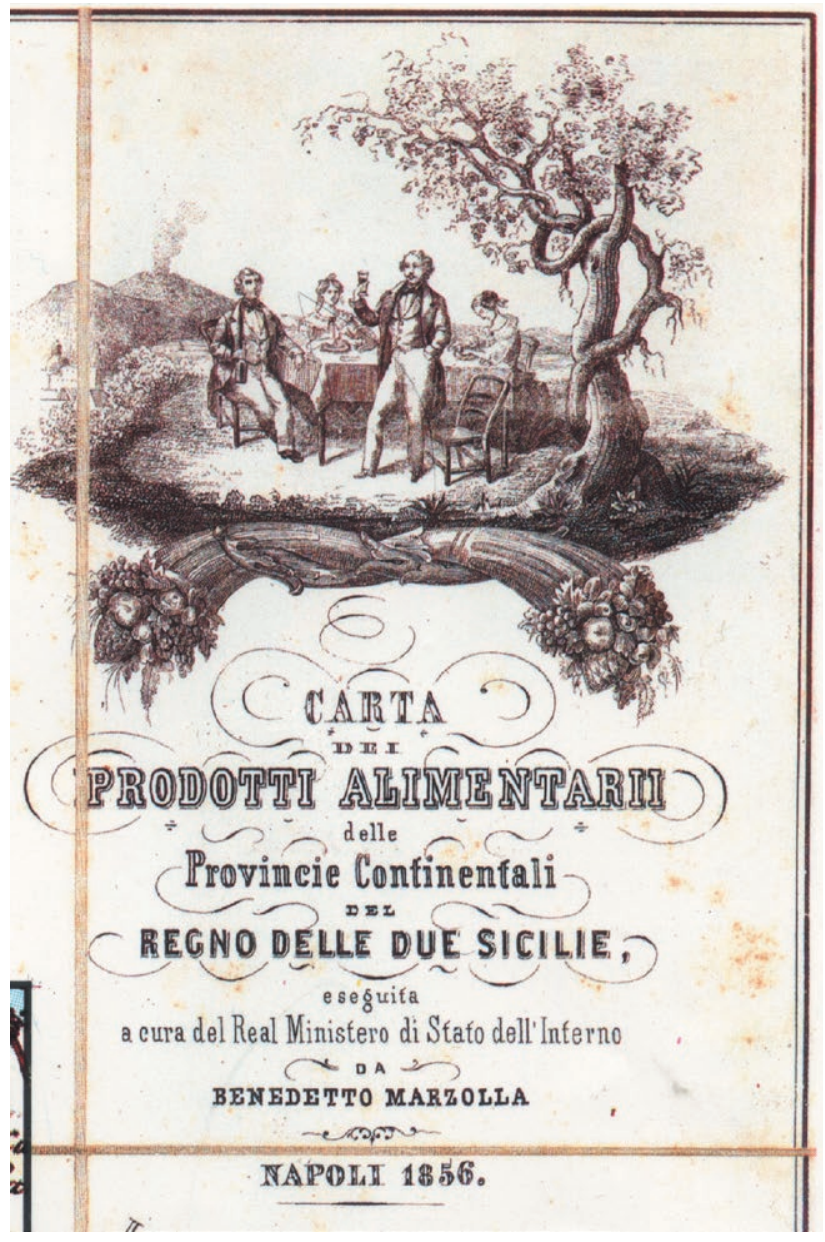

Figura 2. Cartiglio della Carta dei prodotti alimentari.

il servizio e rappresenta la tutela giuridica di un segno (logo) che lo distingue. Proprio l'Italia detiene il primato per numero di denominazioni protette, legato a produzioni agro-alimentari e gastronomiche "tipiche", ma la ratio alla base di questo riconoscimento è diametralmente opposto - almeno nelle origini - da quanto propone il cartografo brindisino.

Recentemente, la normativa per l'individuazione e per il riconoscimento delle denominazioni, nonché le caratteristiche dei disciplinari, ha posto un'evidente attenzione alla tutela dei territori, dei lavoratori e dei consumatori, ma la filosofia della certificazione di qualità affonda le proprie radici nella competitività, nel marketing territoriale e nella proprietà intellettuale, legata alle procedure produttive ${ }^{4}$.

${ }^{4}$ I primi regolamenti sono il Reg. CEE 2081/1992 (Protezione delle indicazioni geografiche e delle denominazioni di origine) e il Reg. Cee 2082/92 (sulle Specialità Tradizionali Garantite), sostituiti nel 2006 


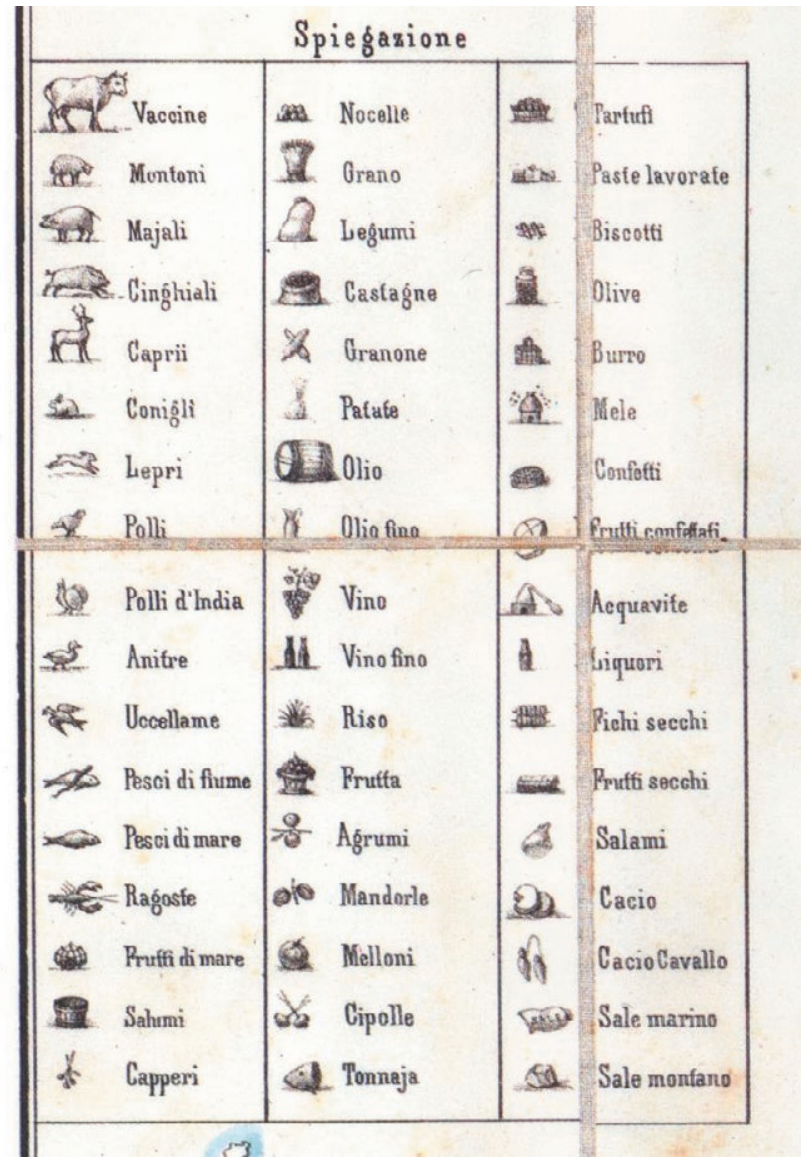

Figura 3. Legenda della Carta dei prodotti alimentari.

Quello che è certo è che delle località legate a dei prodotti alimentari sono divenuti celebri toponimi proprio perché veicolati dalla commercializzazione (e più tardi dalla automazione del processo produttivo) di determinati alimenti. Il binomio luogo-prodotto (si pensi alla pasta di Gragnano, tra tutti) dimostra tutto il potente portato multifunzionale della rappresentazione cartografica di Marzolla che funge da lontano e simbolico raccordo tra valorizzazione, pubblicizzazione e tutela del patrimonio locale (volta all'esaltazione del Regno) e analisi territoriale statistico-scientifica.

rispettivamente dal Reg. CE 510/2006 e dal Reg. CE 509/2006. Tali aspetti normativi sono stati formalizzati nel "Libro Verde sulla qualità dei prodotti agricoli: norma di prodotto, requisiti di produzione e sistemi di qualità" (Com (2008) 641) che ha originato il "Pacchetto Qualità" al quale è seguito il Reg. UE 1151/2012, divenuto il nuovo testo di riferimento disciplinare. Per ciò che riguarda i vini e le bevande spiritose, è stato emanato il Reg. CE 479/2008: dal $1^{\circ}$ agosto dell'anno successivo, infatti, i vini DOP e i vini DOCG sono stati inclusi tra le DOP, mentre i vini IGT nellelenco delle IGP.

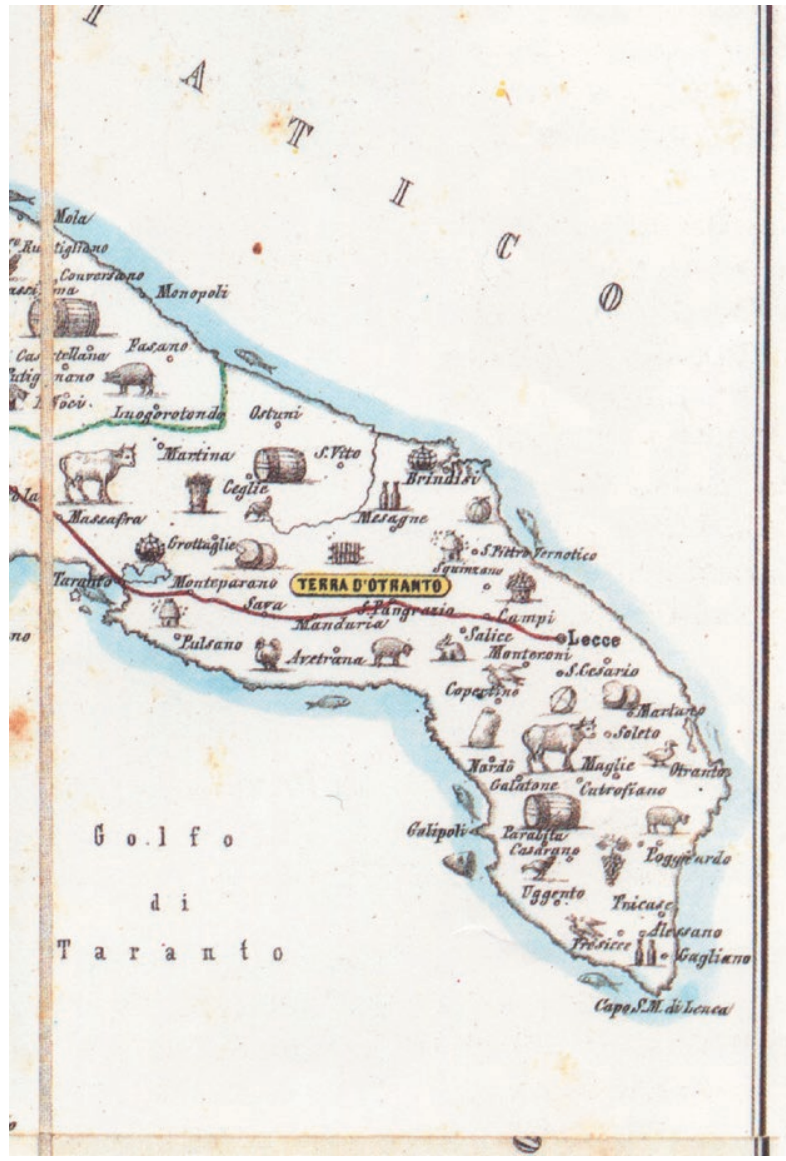

Figura 4. Stralcio della Carta dei prodotti alimentari (Salento- Terra d'Otranto).

\section{Gli studi "preliminari"}

La carta, litografata a quattro colori e che misura $30 \times 40 \mathrm{~cm}$ (scala intorno al milionesimo), consta di una legenda di 51 simboli, illustra nel dettaglio la produzione in alcune delle aree del Mezzogiorno, quale parziale ripresa delle descrizioni che si trovano nelle legende delle carte che compongono la raccolta che Marzolla esegue tra il 1828 e il 1833. Queste ultime sembrano quasi lo studio "preparatorio" alla carta del '56 (le pur dettagliate aree della Sicilia, come detto, non saranno menzionate, mentre nella raccolta si considerano anche le enclave di Pontecorvo ${ }^{5}$

${ }^{5}$ Nel Cenno Storico-Statistico della Città di Pontecorvo, relativa alla carta edita da Marzolla nel 1833, si legge "Nel 1806, allorché il Regno divenne occupato dalle armi francesi, Pontecorvo fu dato in Principato al Maresciallo Bernadotte ora Re di Svezia. Allorché felicemente i Reali Dominii al di qua del faro ritornarono sotto il paterno e legittimo reggimento dei Borboni, nel 1815, tanto il Principato di Pontecorvo, che quello di Benevento furono resi al dominio della Santa Sede ed ora godono dell'amoroso e saggio Governo di Gregorio XVI felicemente regnante". Ponte- 
e Benevento ${ }^{6}$ appartenenti allo Stato Pontificio ${ }^{7}$ escluse poi dalla Carta dei Prodotti). Nella raccolta in effetti, non vengono menzionate soltanto le produzioni agroalimentari, ma si fa riferimento anche a quelle trasformate che - a seconda della carta - vengono definite "prodotti manufatturieri" o "prodotti naturali, industria, fabbriche, manifatture e commercio" e ordinate secondo la catalogazione ufficiale ${ }^{8}$.

Ecco alcuni stralci dalle notizie contenute nei citati Atlanti del Marzolla, "propedeutiche” per l'esecuzione della Carta.

\section{Provincia di Napoli (1829):}

"Napoli [...] fabbriche di cappelli di pelo e di paglia, di coralli, di guanti, di fiori all'uso di Francia, di acciajo, di terraglia, di lava del Vesuvio, di oro, di argento, biscuitterie, di lastre, fogli di stagno per gli specchi, di cotone all'uso inglese e di Lucca. Tessuti di ferro per usi diversi. Castori all'uso di Francia. Bottiglie nere, porcellane benissimo dipinte, di spielle, caratteri per tipografie, carrozze, di seta, di galloni, di bottoni, di cardi, di profumi, d'armi. Nastri, merletti, filo, vetro, ec. ec. [...]. Castellamare (Stabium). Vi è fabbrica di pelli all'uso forestiere. Cantiere per la costruzione de' vascelli. I maccheroni, le paste minute e le acque minerali formano la sua ricchezza. Lettere e Gragnano. Vini leggieri e poco durevoli, ma squisiti. Sorrento. Seta per calze, butirro ed ottime carni. Torre Annunziata. $\mathrm{R}^{\mathrm{e}}$ fabbrica d'armi e Polveriera, Valchiere, Camiere, si lavorano paste d'ogni sorte, di cui si fa esattamente spaccio. In Pozzuoli fabbrica di saponi all'uso di Marsiglia, vini [...]. Procida e Monte. Ottimi vini, abbondante di quaglie, fabbrica di bottoni di fili per biancheria. Ischia. Erbe, frutta, pesci, latte, vini perfettissimi e squisiti. Bagni ed acque minerali. Capri produce legumi, olio, vino, eccellenti frutta e vitelli ricercatissimi. Erba rubbia per le tinte. In maggio abbondanza di quaglie."

Provincia di Principato Ultra (1828):

"Il grano, il granone, il vino sebbene non di ottima qualità, i legumi, la frutta, gli ortaggi, il lino, il canape, sono i suoi prodotti, e particolarmente le castagne e le nocelle di cui con molto profitto abbondano, in spezialità Avellino e Monteforte. Nel territorio di Ariano si contano anche delle acque minerali e delle cave di gesso".

corvo si proclamò parte del Regno di Italia il 2 settembre 1860, subì poi il ritorno delle autorità pontificie, supportate dalle truppe borboniche, per essere infine definitivamente riconquistata dai soldati di Re Vittorio Emanuele II, il 7 dicembre dello stesso anno.

${ }^{6}$ Nella legenda della carta edita da Marzolla nel 1833 si legge "la quale [Benevento] nelle passate vicissitudini d'Europa fu feudo imperiale dal 1807 al 1814 a favore del Principe di Talleyrand. Il Congresso di Vienna del 1815 la rese alla Santa Sede". Il 2 ottobre 1860 sorge un governo provvisorio e il 25 ottobre Benevento diventa una provincia del Regno di Vittorio Emanuele II.

${ }^{7}$ Stato della Chiesa fino al 1815.

${ }^{8}$ Biblioteca Nazionale di Napoli, sez. Lucchesi-Palli I.18.III.8.
Provincia di Bari (1829):

"Le sue produzioni sono egualmente estese in grani, olj, e vini. Vi si raccolgono mandorle in quantità considerevoli da farne vendita all'Estero. Le saporose cipolle e le carrubbe sono due articoli di grande commercio attivo colle Provincie limitrofe. Le sue pastorizie sono anche ragguardevoli e il formaggio soprattutto presso Gravina, ove sono eziandio buonissime razze di cavalli al pari che in Altamura e Conversano. In Andria si raccoglie del buon mele. La bambagia è coltivata nella maggior parte de'paesi. Barletta esporta principalmente grani, sei miglia lungi verso l'Ofanto sono le Grandi Saline di regio conto. Trani [...] vi si fabbrica un moscato gustosissimo [...]. Molfetta è commerciantissima, ha molte fabbriche di funi. Bari ha fabbriche di rosolj [...]. Fabbrica di nitro, sapone, tintorie, concerie, telaj di felpa, tele e fazzoletti ec. ec.".

\section{Provincia di Capitanata (1830):}

"Il grano, le biade, i legumi, il vino sono i principali prodotti di questa Provincia. L'olio abbonda a Lucera, in S. Severo, e per il paese del Gargano, ove si coltivano gli agrumi, e le carrubbe, e si raccoglie mele, pece e manna. Non mancano le varie frutta, ed il littorale gode di una mediocre pescagione".

Delegazione di Benevento (1833):

"Il suolo del Ducato Beneventano è assai fertile, produce ogni sorta di cereali, bovini, vini, olio, e tutte spezie di frutta. Gli ulivi si coltivano con maestria, e si fa grande spaccio di vegetali di ogni genere con le comuni convicine del Regno. La coltura del tabbacco forma la principale industria. I torroni di Benevento sono rinomati ovunque, massimo consumo se ne fa nel SS Natale per Roma ed altre Città. I formaggi ritratti dai bestiami dei suoi territorj sono squisiti, si fa in Benevento gran commercio di bestiame, conta varie fabbriche di cappelli e l'accreditata fabbrica di corde armoniche: è orgogliosa nella perfezione in tutte le arti, e solo può essere dalla Capitale superata”.

\section{Delegazione di Pontecorvo (1833):}

"Vi è della caccia di lepri, caprj, ed anche di cinghiali, sebbene non vi sono boschi [...] e similmente più specie di pennuti, secondo le stagioni. Il detto fiume [Liri] dà della pesca a quegli abitanti e comodo ancora per lo trasporto di più sorte di vettovaglie. [...] Oltre all'agricoltura, e la pastorizia, essi hanno l'industria ancora di nutricare i bachi da seta, che vi fa un buon capo di guadagno. Vi si fabbricano ancora delle funi, e de vasellami di creta, ma ordinarj".

Provincia di Calabria Ultra II (1831):

"Sono produzioni principali della Provincia il grano, il vino, l'olio, il riso, il lino, il canape, il cotone, lo zafferano, la manna, il sale, il mele, la seta, la pastorizia moderatamente trattata, ma non meno rinomati che forti e belli sono i cavalli che nascono in questi luoghi, i quali soprattutto portano il vanto sopra tutti quelli del Regno". 
Provincia di Calabria Citra (1831):

"[...] produce ora grano, olio, vino, lino, canape, riso, zafferano, mele, cotone, seta, manna, sale, legumi, ed ogni sorta di frutti e di prodotti ortalizj. Esporta principalmente abbondanti olj e vini ricercati. Contiene miniere di vario genere, di ferro, di zolfo, e molte cave di alabastro e cristalli di monte. Nutre varie mandrie, e non è quindi scarsa di latticinj. Produce bellissimi cavalli e forti, non che muli ed asini. Vi si fa grande industria di neri ed ottimi salami che in abbondanza si esportano da per tutto. Vi ha pesca e caccia. Si manifatturano con lode le pelli all'uso di forestiere. Non mancano infine le buone fabbriche di cappelli, non che telaj di flanelle, castorini, e tessuti di cotone d'uso comune ec. In Corigliano particolarmente sono mantenute fabbriche di liquorizia, ed un ragguardevole traffico di legname da costruzione per uno della $\mathrm{R}$. Marina.”

Uno degli elementi di questa disamina che più colpisce è l'idea che i vini dell'avellinese siano abbondanti "sebbene non di ottima qualità". Come sappiamo, infatti l'Irpinia è considerata ai nostri giorni la culla delle produzioni vinicole di pregio come il Taurasi, a base di Aglianico, il Fiano di Avellino, ottenuto dall'omonimo vitigno e il Greco di Tufo. Nella stessa area si produce: tra i rossi, oltre all'Aglianico, anche lo Sciascinoso e il Piedirosso; tra i bianchi, oltre al Greco e al Fiano, anche il Coda di volpe e la Falanghina. Neanche nella Carta dei Prodotti sono menzionate queste qualità: l'area di Solopaca e tutta la zona occidentale del Sannio e dell'Irpinia non vengono collegate alle produzioni vitivinicole. Ma sappiamo anche che in passato i vini migliori della Campania erano soltanto quelli derivati da vigneti posti in zone con suoli vulcanici, incluso la Piana Campana coperta dai tufi flegrei.

Per ciò che riguarda la filiera dei prodotti caseari in Campania, a parte quelli generalmente attribuiti all'area della Penisola Sorrentina, appaiono grandi assenti quella di San Giuseppe Vesuviano nonché quella di Capaccio, le quali (caratterizzate dalla profonda evoluzione innovativa), sembrano essere successive alla realizzazione o ai sopralluoghi volti alla compilazione della carta. La produzione del provolone del Monaco (D.O.P. attualmente) ad Agerola e Gragnano ha avuto inizio nel 1850, seguita poi dalla filiera vesuviana dell'Auricchio (oggi il Provolone Valpadana D.O.P. di Cremona) il cui primo caseificio vide la luce nel 1877, mentre le aziende dell'area di Paestum nasceranno soltanto nel primo decennio del XX secolo (Cundari 2018, 72 e ssg.).

Alcuni degli elementi che presenta Marzolla vengono dunque confermati e dettagliati nella Carta che considera queste produzioni tra le "tipiche" scegliendo tra quelle che paiono più significative nell'ambito della trat- tazione e che per comodità e facile comprensione del lettore accorpiamo alle partizioni amministrative contemporanee (Tab. 1).

\section{Il sistema commerciale e doganale del Regno}

Per comprendere al meglio il sistema commerciale della produzione del Regno delle Due Sicilie, non si può non far riferimento al sistema doganale del tempo.

Secondo quanto riportato da Landi (1977) l'Amministrazione dei Dazi Indiretti del Regno fu istituita inizialmente nel $1806^{9}$. Era divisa in quattro sezioni: i dazi nelle dogane di terra e mare; i tributi gravanti sui beni commestibili all'interno del Regno; i proventi dalla vendita del sale; i "diritti riuniti" (come, ad esempio, la lotteria o le bevande alcoliche); inoltre, l'Amministrazione aveva introdotto una contribuzione fondiaria a somma fissa che risparmiava solo le attività agricole di interesse strategi$\mathrm{co}^{10}$. Dal 1814, poi, l'Amministrazione fu ripartita in due rami distinti (dogane e gabelle di consumo; e per i diritti riservati). In seguito, tramite regi decreti del 5 settembre 1815 e del 5 marzo 1816, venne riorganizzato il servizio e furono istituiti diversi trattati di commercio con Spagna, Francia e Inghilterra, in concomitanza all'emanazione della legge di navigazione, varata il 30 luglio $1818^{11}$.

9 “Quanto a' dazi denominati indiretti, ho già esposto nella sezione I di questo capitolo, come il governo nel richiamare alla finanza la riscossione di tutt'i dazi di tal natura, o sotto la denominazione di arredamenti, o sotto quella di passi, piazze e dogane baronali, o di uffici venduti o donati, avesse provveduto alla soddisfazione di tutt'i creditori che o parte di quei dazi possedevano, o avevano assegnamenti sulla rendita loro. Medesimamente a' diciassette giugno e a' sedici agosto 1806 venne istituita una generale amministrazione detta de' dazi indiretti nella quale furono compresi i diritti conosciuti sotto il nome di doganali, gli arredamenti secondo che' gli ho descritti nel capitolo III del precedente libro, la carta bollata, la lotteria, la crociata, il protomedicato, e quanto altro sotto il generico nome d'indiretti tributi potesse appartenere allo stato" (Bianchini 1839,515$)$. Inoltre, per corroborare tale normativa viene varata la legge del 24 febbraio 1809, con si organizza "un sistema d'amministrazione generale e di percezione de' dazi indiretti per le dogane, sali, dazi di consumo e diritti riuniti" (Archivio di Stato di Napoli [ASN], Leggi e decreti, a. 1809, I s., n. 290, 225-291; in Rescigno 2007, 5).

${ }^{10}$ All'interno della gestione "francese", inoltre vi era: il Tesoro reale, la Corte dei Conti, il Banco delle Due Sicilie, la Cassa di Ammortizzazione, la Cassa delle Rendite, la Direzione del Gran Libro, la Direzione delle Contribuzioni dirette, la Direzione dei Dazi di consumo, la Direzione della Gran Dogana di Napoli, la Direzione di Terra di Lavoro e dei Due Principati, l'Amministrazione generale dei Dazi riservati, la Direzione dei Diritti riservati di Napoli, la Regia Zecca delle monete, l'Amministrazione dei Reali Lotti, l'Amministrazione della Registratura e dei Demani, l'Amministrazione generale delle Poste ed, infine, l'Amministrazione generale di Acque e Foreste secondo quanto riportato in Stato degli Impiegati del Ministero Finanze del 1813 (ASN, Ministero Finanze, reg. 522 bis, in Rescigno 2007, 18).

${ }^{11}$ Nonostante il 9 giugno 1815 il Congresso di Vienna avesse sancito il ritorno dei Borbone nel Regno delle Due Sicilie (nato dalla riunificazio- 
Tabella 1. Alcuni dei prodotti e delle località menzionate da Marzolla e le produzioni contemporanee "tipiche"

Regione Elenco attuale delle produzioni tipiche

Campania

Vino: Ischia, Capri, Aversa, Beneventano e Irpinia (zona N), Roccamonfina, Cilento.

Vino fino: Campi Flegrei, Vesuvio.

Paste lavorate: Penisola Sorrentina (Gragnano), Torre Annunziata. Biscotti: Castellammare di Stabia.

Olio: Alto Cilento.

Olio fino: Sorrento, Cilento.

Olive: Gaeta, Itri.

Granturco: Un po' ovunque, nelle vallate aperte.

Nocelle: Avellino, Avella.

Riso: Castellammare ${ }^{\mathrm{a}}$.

Tonnaja: Castellabate.

Burro: Vico.
Formaggi (D.O.P.): Caciocavallo Silano, Provolone del Monaco, Mozzarella di Bufala Campana.

Altri prodotti di origine animale (D.O.P.): Ricotta di Bufala Campana. Oli e grassi (D.O.P.): Cilento, Colline Salernitane, Colline dell'Ufita, penisola Sorrentina, Terre Aurunche.

Ortofrutticoli e cereali (D.O.P.): Cipollotto Nocerino, Fico Bianco del Cilento, Pomodorino del Piennolo del Vesuvio, Pomodoro S. Marzano dell'Agro Sarnese Nocerino, Oliva di Gaeta.

Ortofrutticoli e cereali (I.G.P): Rucola della Piana del Sele, Nocciola di Giffoni, Melannurca Campania, Marrone di Serino/Castagna di Serino, Marrone di Roccadaspide, Limone di Sorrento, Limone Costa d'Amalfi, Castagna di Paestum, Castagna di Montella.

Pasta alimentare (I.G.P): Pasta di Gragnano.

Pesci, molluschi, crostacei freschi (D.O.P.) Colatura di alici di Cetara.

\section{Calabria}

Formaggio (cacio specie caciocavallo): in parecchie contrade specie Cosentino, Sila, Serre.

Cipolle: Tropea.

Tonnaja: Tropea, Pizzo (forse Palmi).

Olio: Molto, ma non fino.

Vino fino: Reggino, molto altrove comune.

Salumi: specie Reggino e Serre, ma anche altrove.

Riso: Valle Crati.

Castagne: Molte, data l'estensione dei oschi.

Api, miele: Pregiato per la selvatichezza montana e collinare, molti fiori.

Cacciagione: Cinghiali, cervi, lepri per la selvatichezza montana. Fichi secchi. Cosenza.

Frutta candita: Reggino (ancor oggi a Bagnara esiste una ditta che produce canditi, torroncini e simili di altissimo pregio, premi internazionali).
Altri prodotti dellallegato I del trattato (spezie, ecc.) e Prodotti di panetteria, pasticceria, confetteria o biscotteria (D.O.P.): Liquirizia di Calabria.

Formaggi (D.OP.): Pecorino Crotonese, Pecorino del Monte Poro, Caciocavallo Silano.

Oli e grassi (D.O.P.): Alto Crotonese, Bruzio, Lametia.

Oli e grassi (I.G.P.): Olio di Calabria.

Oli essenziali (D.O.P.): Bergamotto di Reggio Calabria - Olio essenziale.

Ortofrutticoli e cereali (D.O.P.): Fichi di Cosenza.

Ortofrutticoli e cereali (I.G.P.): Patata della Sila, Limone di Rocca Imperiale, Clementine di Calabria, Cipolla Rossa di Tropea Calabria. Prodotti a base di carne (D.O.P.): Capocollo di Calabria, Pancetta di Calabria, Salsiccia di Calabria, Soppressata di Calabria.

Prodotti di panetteria, pasticceria (I.G.P.): Torrone di Bagnara.

\section{Basilicata}

Vino e Uva: Vulture e Spinosa.

Grano: Alto Materano.

Granone: Vietri di Potenza.

Suini: Pollino, Avigliano e Matera.

Formaggio e cacio: Pollino, Avigliano, Matera e Valle del Bradano.

Prosciutti: Pollino, Avigliano e Matera.

Tacchino: Muro Lucano.

Ovicoltura: Ferrandina.

Castagne: Montemurro.

Legumi: Pescapagano e Stigliano.

Patate: Viggiano.

Uccellame e lepri: Sant'Arcangelo, Pisticci e Buvo.

Cinghiali: Matera.

Bovini: Melfi e Chiaromonte.

Capre: Pomarico.
Formaggi (D.OP.): Caciocavallo Silano, Pecorino di Filiano. Formaggi (I.G.P.): Canestrato di Molitierno.

Ortofrutticoli e cereali (I.G.P.): Lenticchia di Altamura, Fagiolo di Sarconi, Peperoni di Senise.

Ortofrutticoli e cereali (D.O.P.): Fagioli Bianchi di Rotonda, Melanzana Rossa di Rotonda.

Prodotti a base di carne (I.G.P.): Lucanica di Picerno.

Oli e grassi (D.O.P.): Vulture.

Oli e grassi (I.G.P.): Olio Lucano.

Prodotti di panetteria, pasticceria (I.G.P.): Pane di Matera. 
Regione

Abruzzo

Riso: Valle Sangro.

Confetti. Sulmona (ancor oggi rinomati).

Animali selvatici, cacciagione: abbondanti per motivi analoghi alla

Calabria. Si pensi che Pescara, oggi maggiore città, era un piccolo borgo di pescatori.

Liquori: di vecchia tradizione, rinomati quelli da essenze di erbe selvatiche e frutta idem (D’Annunzio, poi, a pagamento, inventò il nome Aurum e la forma della bottiglia).

Vino: Di vario pregio e diffusione.

Grano: Teramo (che ospita ancora oggi un distretto di pastifici), Celano

(dove attualmente si possono reperire "pastifici artigianali"), Antrodoco vicino Amatrice (che attualmente ospita un pastificio) e Palena.
Elenco attuale delle produzioni tipiche

Altri prodotti dell'allegato I del trattato (spezie, ecc.) e Prodotti di panetteria, pasticceria, confetteria o biscotteria (D.O.P.): Zafferano dell'Aquila.

Carni fresche (e frattaglie) (I.G.P.): Agnello del Centro Italia.

Oli e grassi (D.O.P.): Aprutino Pescarese, Colline Teatine, Pretuziano delle Colline Teramane.

Ortofrutticoli e cereali (I.G.P.): Carota dell'Altopiano del Fucino, Patata del Fucino.

Prodotti a base di carne (D.O.P.): Salamini italiani alla cacciatora.

\section{Molise}

Grano: nell'area al confine con l'Abbruzzo e a Campobasso dove anche oggi si concentrano pastifici (es. La Molisana), olio, legumi (anche oggi) salumi, formaggi.
Formaggi (D.OP.): Caciocavallo Silano, Mozzarella di Bufala Campana.

Altri prodotti di origine animale (D.O.P.): Ricotta di Bufala Campana. Oli e grassi (D.O.P.): Olio varietà Molise.

\section{Puglia}

Vaccine, montoni, conigli, polli e polli d'India e suini: Spinazzola, Manduria, Avetrana, Maglie, Poggiardo.

Legumi: Nardò.

Frutti confettati: Lecce.

Frutti di mare: Brindisi, Grottaglie.

Pesci di mare: Paola, Cariati, Barletta, Gallipoli, Taranto, Leuca. Grano: Lucera, Troja, Minervino.

Olio: Gioja, Galatone, S. Vito, Ostuni, Viesti.

Olive: Andria.

Olio fino: Terlizzi, Mesagne, Corato.

Vino e vino fino: Nocera, Poggiardo, Corato, Bari.

Cacio e caciocavallo: Cerignola, Martano, Grottaglie, Foggia.
Formaggi (D.O.P.): Caciocavallo Silano, Canestrato Pugliese, Mozzarella di Bufala Campana.

Formaggi (I.G.P.): Burrata d'Andria.

Altri prodotti di origine animale (D.O.P.): Ricotta di Bufala Campana. Oli e grassi (D.O.P.): Collina di Brindisi, Dauno, Terra d'Otranto, Terra di Bari, Terre Tarentine

Oli e grassi (I.G.P.): Olio di Puglia.

Ortofrutticoli e cereali (D.O.P.): Patata novella di Galatina, La Bella della Daunia.

Ortofrutticoli e cereali (I.G.P.): Arancia del Gargano, Carciofo

Brindisino, Clementine del Golfo di Taranto, Limone Femminello del Gargano, Uva di Puglia, Lenticchia di Altamura.

${ }^{a}$ La coltura del riso, ripresa nel dettaglio nella Carta Catastale di Castellamare (Manzi, 2007a) è, secondo Carrabetta, "un’altra conferma della diffusione residua (all'epoca) della coltura nel Sud. Oltre un terzo del suolo rappresentato in questa mappa è solcato da fossi a coltura risicola: si tratta dell'area più bassa dove si può agevolmente derivare l'acqua dal fiume, con cui i fossati sono in comunicazione" (2003, p. 337).

L’anno successivo venne stipulata una Convenzione doganale, firmata il 7 dicembre, secondo la quale le due enclaves (i ducati di Benevento e Pontecorvo) venivano "assimilati sotto questo rapporto a tutti gli altri Comuni di dominii del governo di Sua Maestà e come tali dovranno in seguito essere considerati" 12 facendo sì che il Regno divenisse nei fatti un'unione doganale che aveva un solo confine terrestre con lo Stato Pontificio ${ }^{13}$.

ne degli antichi regni di Napoli e di Sicilia) l'ordinamento amministrativo del Decennio francese fu sostanzialmente preservato.

${ }^{12}$ Collezione delle leggi e de' decreti reali del Regno delle Due Sicilie, Napoli, Dalla Real Tipografia del Ministero di Stato della Cancelleria Generale, anno 1820. Semestre I, 65.

${ }^{13}$ In effetti, nella legenda della carta raffigurante la Delegazione di Benevento del 1833 si legge "La Dogana di Benevento messa a livello con quelle del Regno può considerarsi di $2^{\text {a }}$ classe".
Il sistema dei Dazi nel Regno ebbe poi una svolta a partire dal 1823, in seguito all'applicazione della strategia dell'allora ministro dell'economia, Luigi De' Medici (Ciccolella 2012). La programmazione economica era sostanzialmente volta alla promozione dell'industria interna per creare sbocchi per i produttori agricoli:

"Le nostre derrate deggiono consumarsi. Quando gli stati vicini credono, che non convenga ai loro interessi di acquistare i nostri cereali, le nostre lane, i nostri olii, ed i nostri cotoni, e le altre derrate, ci obbligano a doverle impiegare in fabbriche di panni, in saponi, in lavori di cotone ed impedire siffatte di loro provvenienze"14.

\footnotetext{
${ }^{14}$ ASN, Archivio Borbone, fs. 669, Memoria su le convenzioni che hanno
} 
In seguito, con Real decreto del 20 novembre 1824 furono approvate tariffe sui diritti di importazione ed esportazione che, secondo quanto individuato da Lepre $(1969,563)$, appaiono avere un carattere "difensivo"; il 13 aprile $1826 \mathrm{fu}$ fissata l'organizzazione della Direzione generale de' Reali Domini al di qua del Faro ${ }^{15}$, mentre con la legge delle Dogane emanata il 19 giugno 1826 e il Reale Decreto del 9 gennaio 1827 fu organizzata anche la regolamentazione dei dazi e la risoluzione dei loro contenziosi (Mastriani 1842, VII).

Queste ultime normative diedero un ulteriore stretta al contrabbando che affliggeva il territorio, soprattutto per i prodotti quali sale, tabacchi e caffè (proveniente da Trieste), i prodotti finiti di origine inglese e francese ed in particolare i tessili; inoltre, sancirono l'abolizione del sistema dei dazi cosiddetti ad valorem ${ }^{16}$ portando all'abrogazione della precedente pratica delle tare (Nicali 1997); infine contribuirono a riconfigurare l'ordinamento dell'Amministrazione dandole il compito di sovrintendere alle dogane, ai commerci, ai dazi erariali per i beni di consumo e quelli sottoposti a monopolio o privativa (sale, tabacchi, carte da gioco, polveri da sparo, neve). Le politiche di De' Medici diedero vita a una stagione di relazioni commerciali tra il regno e la Spagna, la Francia e l'Inghilterra ${ }^{17}$.

abolito il privilegio delle bandiere franche, e sui decreti di Sua Maestà / Dio Guardi/ per favorire la Marina mercantile, e le interne produzioni, s.d. ma settembre-ottobre 1823. La memoria è anonima ma fu redatta dal capo ripartimento del ministero delle Finanze su ordine e secondo precise istruzioni del ministro de' Medici (cfr. ASN, Ministero delle Finanze, fs. 4712, f.lo 8963, Memoria da farsi su gli ultimi decreti doganali in forma didascalica, 14 settembre 1823; in Ciccolella 2012, 407).

${ }^{15}$ Il "faro" a cui si fa riferimento è quello della città di Messina. Con la legge dell'8 agosto $1806 \mathrm{n}$. 132 venne riformata la divisione ed amministrazione delle provincie del Regno, secondo il modello francese. In seguito al decreto del 4 maggio 1811 il Regno fu decisa "una nuova circoscrizione delle quattordici provincie del Regno di Napoli". Ai Domini "al di qua del Faro" appartenevano: la Provincia di Napoli, Terra di Lavoro, il Principato Citeriore, il Principato Ulteriore o Ultra, la Basilicata, Capitanata, Terra di Bari, Terra d'Otranto, la Calabria Citeriore, la Calabria Ulteriore II, la Calabria Ulteriore I, il Contado di Molise, Abruzzo Citeriore, Abruzzo Ulteriore II, Abruzzo Ulteriore I. I Domini "al di là del Faro", seppur investiti da diverse riforme amministrative sino al 1837, erano: la Provincia di Palermo, la Provincia di Messina, la Provincia di Grigenti, la Provincia di Siracusa, la Provincia di Trapani, la Provincia di Caltanissetta; secondo quanto riportato nell'Almanacco (Mariani 1859, 275), il Regno delle Due Sicilie nel 1856 contava una popolazione di circa 9 milioni di individui che abitavano le 22 succitate Provincie, con 77 Distretti, 720 Circondari e 2.210 comuni.

${ }^{16}$ Cioè quelli il cui importo è stabilito in proporzione al prezzo della merce.

${ }^{17}$ Lo storico Graziani (1960) ha pubblicato una cospicua documentazione statistica sul commercio estero (importazioni e esportazioni) delle Due Sicilie dal 1832 al 1855 in considerazione di vari "gruppi merceologici”. Tale analisi testimonia un basso tasso di esportazioni dello Stato se confrontato agli altri Paesi europei coevi (e solamente per beni specifici, come lolio di oliva dalla Terra di Bari o lo zolfo dalle zone di
Ed è dunque proprio in considerazione di tali analisi storiografiche ed economiche che appare cruciale la Carta indicante il servizio dell'Amministrazione Generale de' dazj indiretti de' Reali Domini al di qua del Faro (litografata a tenui colori e di $55 \times 43 \mathrm{~cm}$ a scala di circa 1:2.000.000), editata dal cartografo brindisino e compilata dal coevo campano Gaetano Valentino, il 30 settembre 1830 presso la Reale Litografia Militare ${ }^{18}$, ventisei anni prima dell'edizione della Carta dei Prodotti alimentari nonché anno dell'ascesa al trono di Ferdinando II.

La carta che - come si legge in legenda - indica il servizio dell'Amministrazione con una "Ricapitolazione numerica" 19 delle officine di dazi indiretti, di consumi, dogane, fondaci, ispezioni, finanze, posti di guardia e servizio di mare, presenta un cartiglio in cui si indicano le distanze da $\operatorname{Napoli}^{20}$ (città del "Muro finanziere"21) e un francobollo con una specifica riguardante "Pe" Dazj di consumo"22. Nella legenda, innanzitutto, viene riportata la classificazione delle 135 dogane: se ne contano 21 di $1^{\text {a }}$ classe (di cui 14 marittime incluso il porto franco di Messina, 7 di frontiera), 54 di $2^{a}$ classe (31 marittime e 23 di frontiera ${ }^{23}$ ) e 60 di $3^{\text {a }}$ classe (50 semplici dogane di mare, 9 dogane e fondaci, 1 di sola visita e transito ${ }^{24}$ ).

Enna e Caltanissetta); la rassegna dimostra inoltre che l'economia del Regno fosse basata essenzialmente sull'agricoltura e che l'industria fosse solo embrionale. Inoltre, la polarizzazione creata dalla capitale andava a discapito dell'entroterra del Mezzogiorno continentale.

${ }^{18}$ BNN, sez. Lucchesi-Palli I.18.III.8 (2).

${ }^{19}$ Cosa che, tra l'altro, testimonia il côté statistico-quantitativo di Marzolla.

${ }^{20}$ Per la gestione della Dogana del porto di Napoli, dapprima venne istituita una "scala franca" (ovvero unarea esente dai dazi), abolita nel 1824 e sostituita da un vero e proprio deposito, con termini e dazi particolari; le dogane atte alla riscossione dei dazi erano sette: Napoli, Lecce, Bari, Reggio, Chieti, Foggia, Catanzaro e Cosenza. La Gran Dogana di Napoli, il cui edificio fu realizzato da Stefano Gasse nel 1828, gestiva addirittura il 75\% del gettito dei diritti di entrata del Regno (Graziani 1960).

${ }^{21} \mathrm{Il}$ "muro finanziere", progettato da Gasse e lungo circa $20 \mathrm{~km}$, dalla Maddalena a Posillipo, e voluto da Ferdinando I nel 1824, fu una imponente struttura doganale, edificata con precisi scopi politici e finanziari: ne facevano parte le barriere del ponte della Maddalena, di Poggioreale, di Capodichino e il "cavone" di Miano (Pignatelli 2006). Questo riprende, fondamentalmente, l'antica linea doganale formata dalle sbarre dei tempi aragonesi passante per il Ponte della Maddalena e Capodichino (Reccia 2013,39) come è ben visibile nella cartografia di Buccaro (1828).

${ }^{22}$ A gestire il gettito generato dai dazi di consumo era lo Stato: parte di questo era destinato al fabbisogno finanziario della capitale. Sul tema si veda Ostuni (1992).

23 "Compreso Montesicuro chè dogana di terra e di mare. Si nota che le dogane di Portello, Martinsicuro e Castelluccio sebbene siano di $1^{\text {a }}$ classe nulladimeno per le operazioni rispettive che fanno per le dogane di Giulia, Fondi ed Isola vengono a considerarsi di $2^{a}$ classe".

24 "Le controscritte dogane unite à fondaci de' generi di privativa sono le seguenti: Ponza, Ventotene, Ischia, Agropoli, Capitello, Scalea, Melito, Cirò, e Bianco". 
Si presentano poi i fondaci, in numero di $85^{25}$. Inoltre, all'interno della macrocategoria dei fondaci si distinguono: 1 Deposito, 14 Depositi e Fondaci, 61 Fondaci, 9 Fondaci uniti alle Dogane ${ }^{26}$. Vi sono poi menzionate 54 Officine de' Dazj di consumo: 4 di $1^{\text {a }}$ classe, 4 di $2^{\text {a }}, 14$ di $3^{\mathrm{a}}$, 11 di $4^{\mathrm{a}}, 21$ di $5^{\mathrm{a} 27}$.

Si enumera poi il Gran Fondaco de' Tabacchi di Napoli e il Fondaco della Neve di Napoli. Ci sono poi 8 Ispezioni $^{28}$, 53 Controlli $^{29}$, 35 Controlli sedentarj ${ }^{30}$, 220 Tenezie $^{31}$, 452 Posti $^{32}$, 4 Divisioni di mare, ossia Comandanti di Golette ${ }^{33}$, 4 Scorridoje, 64 Castaudelle, 15 Gozzi $^{34}$.

25 "I suddetti fondaci sono provveduti nel modo seguente: Dalle Saline di Trapani: Napoli, Salerno, Gaeta, Reggio, Castellamare, Roccella, Melito, Bianco, Pisciotta, Agropoli, Capitello, Bagnara. Da Napoli provveduto da Trapani: Ischia, Ponza, Ventotene, Caserta, Capua, Nola, Sessa, Arpino, S. Germano, Piedimonte, Avellino, Montesarchio, Paduli, Campobasso, Isernia, Casteldisangro. Da Salerno prov.to da Trapani: S. Angelo de' Lombardi, Valva, Auletta, Sala, Potenza. Da Barletta per la via di mare sino ad Ortona, e da Altamura a Foggia per la via di terra: Barletta, Bari, Mola, Molfetta, Monopoli, Brindisi, Gallipoli, Lecce, Otranto, Taranto, Montalbano, Rodi, Termoli, Manfredonia, Giulia, Vasto, Pescara, Ortona, Altamura, Venosa, Avigliano, Foggia. Da Pescara prov.to da Barletta: Lucera, Ariano, Chieti, Penne, Solmona, Aquila, Antrodoco, Avezzano. Da Fondaci prov.ti da Barletta: Larino, Riccia, Manduria, Teramo, Lanciano. Dalla Salina di Altomonte: Torre, Cerchiara, Belvedere, Moliterno, Cosenza, Scalea, Lungro, Rossano, Castrovillari. Da Belvedere prov.to da Altomonte: Amantea, Nicastro, Pizzo, Tropea. Da Torre Cerchiara prov.to da Altomonte: Cirò, Cotrone, Catanzaro, Soverato, S. Severina, il quale si provvede da Cotrone".

26 "Sono quelli di sopra descritti", e cioè Ponza, Ventotene, Ischia, Agropoli, Capitello, Scalea, Melito, Cirò e Bianco.

27 "Questa classificazione riguardante i dazj di consumo è a norma del Decreto del 13 aprile 1826, e di altre ministeriali disposizioni ma la medesima è variabile, a misura che il muro finanziere va a compiersi".

28 "Per la vigilanza del servizio lungo le linee di frontiera di terra e di mare. Esse sono $1^{\text {a }}$ Ispezione: da Canneto a Capo Campanella. $2^{\mathrm{a}}$ : da Campanella a Castrocuccaro. $3^{\mathrm{a}}$ : da Castrocuccaro a fiume Mesino. $4^{\mathrm{a}}$ : da Mesino a Crucoli. 5a: da Crucoli a Leuca. $6^{a}$ : da Leuca a Fortore. $7^{\mathrm{a}}$ : da Fortore a Pescara. $8^{\mathrm{a}}$ : da Pescara a Fondi per la frontiera di terra. Si avverte che gli Ispettori sono 13, Decreto de' 13 Aprile 1826. Gli altri cinque sono destinati in Napoli, a norma dell'art. 80 del citato Decreto". 29 "I Controlori nominati sono 64. Si avverte che sebbene i Controlli sono 53 corrispondenti al numero de' Distretti R. ${ }^{\text {li }}$ Dominii al di qua del faro, gli altri 11 Controlori servono nella Gran Dogana, ne' Consumi, ec.".

30 "Quest' impiegati servono appunto ne' luoghi designati agli art. 97,99, 100 e 102 del Decreto de' 13 Aprile 1826".

31 "I Tenenti nominati sono 153 nulladimeno le rimanenti 67 tenenzi e sono occupate da forieri della forza attiva che fanno lo stesso servizio dei tenenti”"

32 "Oltre i posti delle polveriere che sono in diverse province del Regno ed i qualisono guardati giusta gli ordini Sovrani, ed esclusi quelli della Dipendenza de' dazj di Consumo, i quali a misura che il muro finanziere va compiendosi, il num. ${ }^{\circ}$ de suoi posti sarà adattato secondo le circostanze locali e come meglio converrà agli interessi del Real Tesoro".

33 "La $1^{\text {a }}$ Divisione comprende da Canneto a Licosa. La 2 da Licosa al Faro di Messina, la $3^{a}$ dal Faro al promontorio di Leuca compreso il posto di Corsaro. E la 4 a fino al Tronto".

34 "I gozzi sono divisi, cioè 9 al servizio della Gran Dogana, ed i rimanenti ne luoghi ove si trova il segno di convenzione", un'ancora semplice a metà.

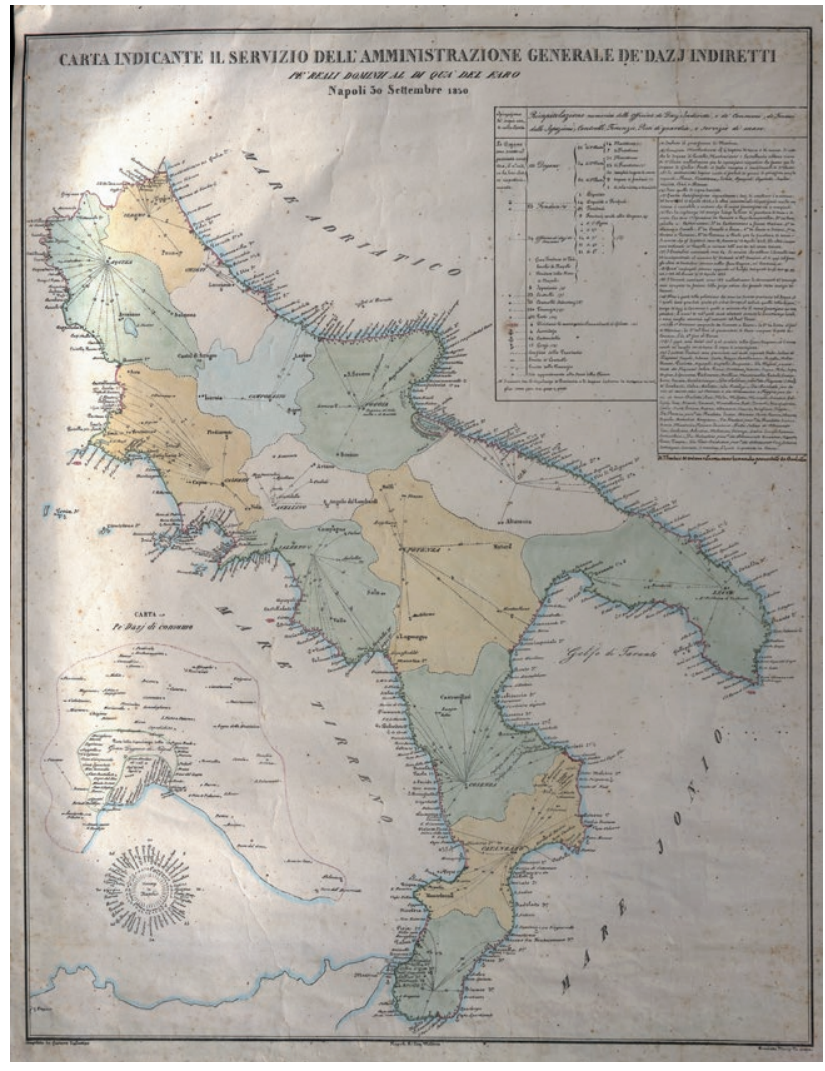

Figura 5. Carta dei Dazi di Marzolla e Valentino, Napoli, 1830. Biblioteca Nazionale di Napoli. Su concessione del Ministero per i Beni e le Attività Culturali e per il Turismo. (C) Biblioteca Nazionale di Napoli. Autorizzazione del 2 marzo 2021- 337-P.

Tale tassonomia trova eco nelle legende della raccolta delle carte del cartografo brindisino già menzionate, alla voce "Dogane della Provincia, giusta la legge organica del 19 giugno 1926".

\section{Per la Provincia di Napoli (1829):}

"Napoli e Castellamare dogane di $1^{a}$ classe, cioè d'importazione, esportazione e cabotaggio. Pozzuoli dogana di $2^{\circ}$ classe, cioè di esportazione e cabotaggio. Ventotene, Ischia, Forio, Lacco, Procida, Granatello, Torre del Greco, Torre dell'Annunziata, Vico, Piano di Sorrento, Massa e Capri dogane di $3^{\circ}$ classe, cioè di semplice cabotaggio ossia di commercio interno".

Per la Provincia di Bari (1829):

"Bari e Molfetta dogane di $1^{a}$ classe. Monopoli, Mola, Bisceglie, Trani, Barletta, dogane di $2^{a}$ classe. S. Vito di Polignano e Giovinazzo, dogane di $3^{\mathrm{a}}$ classe".

\section{Per la Provincia di Capitanata (1830):}

"Manfredonia dogana di $1^{\text {a }}$ classe. Rodi di $2^{\text {a }}$ classe. Viesti, Peschici, Fortore dogane di $2^{a}$ classe". 
Per quella di Calabria Ultra II (1831):

"Dogane di $1^{\text {a }}$ classe sono Cotrone e Pizzo; di $2^{\text {a }}$ classe sono Catanzaro, Soverato, Tropea, Nicastro, di $3^{\text {a }}$ classe sono Melissa, Squillace, Badolato, Nocera”.

La produzione del Marzolla è in genere abbastanza nota agli specialisti, come dimostra ancora una volta l'estesa bibliografia di questo lavoro. Della "popolarità" recente acquisita dalla Carta dei Prodotti alimentari si dice in queste note, e comunque l'uso perdurato anche dopo l'Unità italiana degli atlanti delle province meridionali sia continentali che insulari, attesta la funzione di "supplenza" che la cartografia marzolliana, peraltro di buona qualità, ricoprì in passato, in attesa di rappresentazioni topografiche ufficiali che coprissero l'intero territorio. Queste, comunque, furono a scala topografica e generali, mentre le province di Marzolla, con il tipico, ricco contorno di note, dati e commenti, assumono in qualche modo pure una funzione tematica.

La carta tematica dei Dazi pare sinora sfuggita all'attenzione dei ricercatori del ramo storico-cartografico e qui se ne abbozza un primo commento, anche perché in qualche modo è un'opera propedeutica alla più matura Carta dei Prodotti alimentari.

Probabilmente è stata ignorata dai commentatori, anche per la puntigliosità utilissima degli elenchi della produzione marzolliana, perché essa si trova sì presso la Biblioteca Nazionale di Napoli, ma non nelle sezioni in cui ci si aspetterebbe, ad esempio Provinciale, Palatina, Napoletana, Consultazione, ma nella collezione Lucchesi Palli in gran parte dedicata al teatro allo spettacolo e ad arti analoghe. Una delle curiosità di questa collezione, la cui vicenda si può conoscere dal documentato saggio dell'antica direttrice della Nazionale, Guerriera Guerrieri, è quella delle schede di consultazione scritte da Salvatore Di Giacomo che la diresse nella prima parte del Novecento. Comunque, questa carta daziaria, opera "di servizio" redatta quando già Marzolla era un apprezzato membro del ROT, aggiunge soltanto un ulteriore tassello al ricco mosaico della produzione marzolliana, tra le più importanti in Italia verso la metà del secolo XIX.

Secondo quanto riportato dallo storico Antonio Farinelli (2020), l'argomento delle dogane e dei dazi non può essere avulso dal contesto degli ordinamenti politici, amministrativi ed economici del Regno. In effetti, dopo la Restaurazione, i Borbone scelsero di adottare i principi mercantilistici agevolando le esportazioni, riducendo i dazi sulle materie prime necessarie alle industrie del Regno, almeno fino al 1845 quando invece la politica economica si orienterà verso un più spiccato liberismo (De Matteo 1982, 12). Testimonianza di tale contesto economico è la costruzione sotto il regno di Francesco I del ponte detto "de' Gigli della Dogana" che scavalcava l'alveo di Pollena alla foce, realizzato dall'architetto Colella ${ }^{35}$, e di quello che fu definito il "Palazzo delle Finanze" che oggi è il Municipio, quadrilatero che

"occupa duecentoquindicimila palmi quadrati, ha ottocento stanze e quaranta corridoi. Il vestibolo è decorato delle statue marmoree di Ruggiero I il Normanno, di Federico II di Svezia, di Ferdinando I di Borbone e di Francesco I di Napoli. In quel palazzo fu impiantata la Borsa de' cambii in una vasta sala; nella quale si ammira la statua marmorea di Flavio Gioja di Amalfi, inventore della Bussola nautica, e gloria di queste belle contrade italiane" (Buttà 1877, cap. I).

Come è noto, attraverso la politica finanziaria di Ferdinando II, si vollero andare ad appianare i deficit che si erano formati nel terzo decennio dell'800 che si colmeranno a partire dal ' 45 (Scirocco 1986): sarà proprio con decreto dell'11 gennaio 1831 che il Re rivedrà $\mathrm{i}$ bilanci delle forze armate e dei diversi dicasteri. Inoltre, ridurrà di circa la metà il dazio sul macino che era stato precedentemente imposto nel $1826^{36}$

"sgravando il popolo di circa settecentomila ducati annui ed abolì quello della carne, del vino e delle privative del tabacco in Sicilia. Abolì eziandio le reali cacce di Persano, di Venafro, di Mondragone, Calvi e Vallo; fece altre economie sopra i siti reali di Capodimonte e di Licola; e restituì ai proprietari, per coltivarle, le terre tenute in affitto per uso di caccia reale" (Buttà 1877, cap. III).

In seguito alla diminuzione dei prezzi sul mercato internazionale, attraverso i decreti del ' 42 , ' 45 e ' 46 , il Re andò ad incidere inoltre sulle tariffe dei dazi di importazione e - come detto - stipulò trattati commerciali con la Francia e l'Inghilterra. Dal '48 poi, il dazio sul macinato sarà completamente abolito sul continente (mentre per la Sicilia la tassazione restava di 300.000 ducati), insieme alla riduzione di un terzo di quello sul sale. Tale politica venne corroborata dall'affermazione delle Camere di Commercio: Ferdinando, non abrogando il sistema francese, si limitò ad assumere le normative riformate nel corpo delle leggi borboniche. La Camera di Napoli, infatti, venne formalmente istituita con regio decreto del

\footnotetext{
${ }^{35} \mathrm{Nel} 1828$ Gasse realizzò a ridosso di questo ponte due barriere doganali: l'Officina della Gabella e l'Officina del Mercato Vaccino. La presenza di gigli di mare nella zona e la presenza della dogana ne hanno creato il nome. Tale ponte oggi non esiste più, bensì il toponimo rimane presso via Marina dei Gigli nel quartiere di San Giovanni a Teduccio.

${ }^{36}$ Francesco I, con decreto del 28 maggio 1826, aveva imposto nei domini continentali il dazio sulla macinazione dei grani. Per la Sicilia, invece il regime fiscale era diverso ed era presente sin dal 1824.
} 
1817 sotto il nome di "Camera consultiva di commercio": si occupava dell'organizzazione di una Borsa, vigilava dall'esecuzione delle leggi contro il contrabbando. Vennero istituite, poi, altre Camere a Messina ${ }^{37}$, a Palermo ${ }^{38}$, a Foggia $^{39}$, a Bari ${ }^{40}$ e a Catania ${ }^{41}$ (Bidischini, Musci 1996, XXV-XXVI).

Come detto, la Guardia dei Dazi Indiretti non subisce trasformazioni con la Restaurazione e rimane l'organismo di controllo fiscale sulle imposte; l'organizzazione, dunque, avviene su base provinciale con Direttori che dipendono dalle due diverse Direzioni Generali dei dazi diretti ed indiretti. Le Guardie dei Dazi Indiretti (composte da controllori/capitani, tenenti, brigadieri, forieri, guardie, marinai e piloti), oltre ad essere nominate dal Direttore Generale, assumono funzioni di polizia giudiziaria proprio per reprimere il contrabbando e ogni violazione, secondo quanto stabilito dal decreto del 27 gennaio $1818^{42}$; negli anni tra il 1827 ed il 1832, vennero edificate nuove linee di vigilanza doganale, controllate dalle Guardie dei Dazi Indiretti ${ }^{43}$ (Pignatelli 2006, 51-59). Dal 1835, poi, specifici corpi vennero assegnati per la tutela del settore della privativa dei tabacchi $^{44}$ (Oliva 1986, 323-326). Tale sistema, che vede fondamentalmente la propria genesi nel decennio francese, ha permesso la definizione di un percorso finalizzato all'accentramento fiscale e di controllo che fa del capillare organismo dei dazi e delle dogane un tipico esempio di state-building.

Questa Carta dei Dazi si aggiunge all'imponente produzione cartografica napoletana dalla fine del Settecento all'Unità, come vari studi recenti hanno messo in evidenza. Qui ricordiamo solo che il Deposito Topografico, una delle prime istituzioni cartografiche di stato in Europa, fu inizialmente fondato nel 1781 nei pressi della piazzetta Rosario di Palazzo (nei Quartieri Spagnoli alle spalle del teatro "Augusteo") sotto la direzione del cartografo Giovanni Antonio Rizzi Zannoni, autore del famoso Atlante Geografico del Regno di Napoli, in 32 fogli, completato nel 1812. Il Deposito, con la Restaurazione, fu poi trasferito a via Pizzofalcone con il nome di Reale Officio Topografico (ROT) con decreto del 21 dicembre 1815 quando compare per la prima volta l'istituzione di "una pubblica biblioteca militare per uso degli

\footnotetext{
${ }^{37}$ R.D. 20 ottobre 1818.

${ }^{38}$ R.D. 27 marzo 1849.

${ }^{39}$ R.D. 12 aprile 1820.

${ }^{40}$ R.D. 27 marzo 1849.

${ }^{41}$ R.D. 26 ottobre 1852.

${ }^{42}$ Decreto 27 gennaio 1818, n. 1087, per "Lappalto de' generi di privativa in varie provincie de "dominj di qua del Faro".

${ }^{43}$ Con il decreto 9 gennaio 1827, n. 1219.

${ }^{44}$ Circolare 18 giugno 1836 sulle "Formalità da eseguirsi nelle visite domiciliari pe'l ramo de'dazj indiretti".
}

ufficiali dell'armata" (art. 7); inoltre, con decreto del 22 gennaio 1817 Ferdinando I modificò l'organizzazione del Deposito, scindendolo in due aree: l'Officio Topografico e il Deposito della Guerra. Richiamato Visconti, per la seconda volta a capo dell'istituzione dal 1835 , si diede un grande impulso ai lavori geodetici e all'edizione della carta generale del Regno, inizialmente proposta nel 1814, ma poi accantonata in quanto

"l’interesse per lo sviluppo economico dell'intero Regno [...] e l'attenzione nei confronti dei problemi nei quali si dibattevano le amministrazioni centrali e periferiche a causa della mancanza di cartografie, erano pressoché nulli nel sovrano" (Valerio 1993, 279).

Marzolla operò a lungo in tale istituzione, proprio nella sede di Pizzofalcone. Nel 1855 il ROT era costituito da una biblioteca, un gabinetto di macchine e strumenti ed osservatorio, lavori interni (disegni e incisioni); tipografia, calcografia, litografia e modelli di fortezze su grande scala; sezione topografica di Palermo ed infine da lavori geodetici e topografici. Il governo del Regno d'Italia provvide ad emanare uno specifico decreto (del 4 agosto 1861) con cui dispose la sopravvivenza temporanea dell'Officio topografico napoletano, come Sezione separata dell'Ufficio Superiore dello Stato Maggiore (Guarducci 2006, 45) fino alla sua chiusura, decretata il $1^{\circ}$ novembre 1879 (Cantile 2007, 39; Manzi 2018).

\section{La Guida Gastronomica TCI e due Atlanti tematici}

Nel 1931 il Touring Club Italiano pubblicò una Guida gastronomica d'Italia in cui si enumerano e commentano, secondo una divisione regionale e provinciale, le specialità della tradizione alimentare italiana e i prodotti relativi, vini inclusi. Dalla carta di Marzolla sono passati poco più di settant'anni e la società degli anni Trenta del Novecento era cronologicamente circa a metà strada tra l'Ottocento marzolliano e oggi. Ma gli usi sono rapidamente evoluti nei decenni recenti e alcuni prodotti da generi di largo consumo sono divenuti di nicchia, quali specialità ancora esistenti, ma di alto prezzo e di ristretta produzione. Non tutto, certo. Comunque, la forte identità locale di una serie di prodotti resiste e un parallelo tra la Guida del TCI e la carta è possibile e accettabile, forse più che la forzata attualizzazione dei prodotti meridionali ottocenteschi secondo criteri di protezione e promozione DOC, IGP, DOCG e simili. Anche l'unificazione politica dell'Italia ebbe un peso consistente, perché i traffici si intensificarono, le aree settentrionali godettero di varie protezioni daziarie, di incentivi pubblici per 

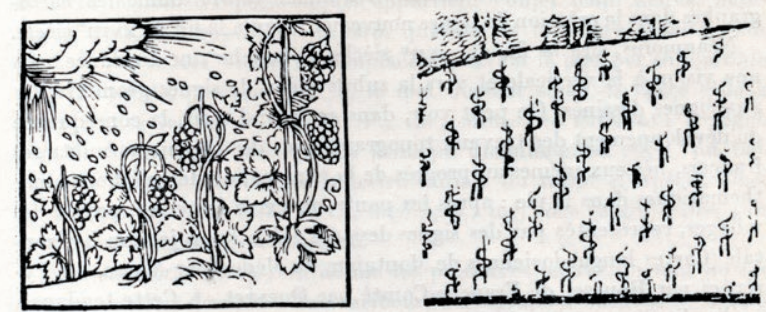

Figura 6. Simboli cartografici per la rappresentazione del vigneto (De Dainville 1964, 327).

stimolare l'industrializzazione delle regioni più prossime al confine terrestre e quindi alla concorrenza europea. Nella Guida del TCI comunque, si nota una certa ammirazione per alcune cucine regionali del Sud, soprattutto quella della Campania (che in gran misura viene identificata con Napoli), della Puglia e della Sicilia. Guarda caso, le tre regioni "forti" dell'agricoltura dell'antico Regno, secondo gli studiosi pre-unitari.

In Campania, nella Guida TCI primeggiano la pasta, il burro pregiato sorrentino (non si scandalizzino i cultori di luoghi comuni sul Mezzogiorno, ancor oggi in Campania perdura la tradizione di latticini e derivati del latte eccellenti, sia latte vaccino che bufalino), le carni bovine del Beneventano, le verdure, la frutta. Per i vini, la Guida non si discosta molto da Marzolla: Capri, Aversa, e le terre vulcaniche di Ischia, Vesuvio e Campi Flegrei.

Un elenco pedissequo delle analogie tra la Guida TCI e la carta di Marzolla per tutte le regioni confermerebbe soltanto che il cartografo era stato ben informato nel suo lavoro, ma annoierebbe il lettore, che rimandiamo all'interessante volumetto, peraltro ristampato dal TCI in anastatica pochi anni fa. Anche una scorsa alle Tavole 68, 69, 71 e 73 dell'Atlante Tematico d'Italia TCICNR è istruttiva per comprendere come la carta alimentare del 1856 fosse ben più ricca di notizie e foriera di ragionamenti geostorici e geoeconomici di quanto la sua linearità espressiva faccia credere. I prodotti cartografici di qualità devono comunicare immediatamente il messaggio informativo, altrimenti non sono cartografia, ma dati statistici confusamente sparsi su un piano ampio, anziché elencati in tabelle o grafici.

Andando ancora indietro nel tempo, si potrebbe consigliare uno sguardo esteso ad alcune tavole dell'Atlante fisico-economico d'Italia diretto da Giotto Dainelli e pubblicato nel 1940 dal Touring Club Italiano, in effetti un antesignano dell'Atlante Tematico apparso mezzo secolo dopo. Sia il fisico-economico che il tematico, furono concepiti come importanti opere semi-ufficiali, nel senso di supplire all'assenza in Italia di un atlante "nazionale", presente invece in molti paesi del mondo.

La Sicilia non rientra in questa disamina, perché Marzolla non estese la sua carta tematica all'isola. In questo seguì in qualche modo la tendenza di tutta la produzione cartografica ufficiale o semi-ufficiale napoletana, dove l'intenzione di far seguire la Sicilia al Continente raramente si concretizzava. La Sicilia scontava come una "condanna", per essere stata raffigurata in una carta famosa redatta con criteri abbastanza all'avanguardia per i tempi, prima della parte continentale del Regno: la carta di Samuele di Schmettau (Manzi 2009a).

Un altro motivo probabilmente risiede nell' uso dei dati del Ministero dell'Interno, forse non abbastanza attendibili per la Sicilia, dove, nonostante la sostanziale unificazione amministrativa con l'accorpamento nel Regno delle Due Sicilie dopo il Congresso di Vienna, perdurava una sorta di autonomia locale. Ci ripromettiamo di approfondire questo ramo d'indagine in un vicino futuro.

Tuttavia Marzolla aveva raffigurato le 7 province isolane nei suoi atlanti, con i medesimi criteri di quelle continentali, e quindi avrebbe potuto utilizzare gli elementi indicativi dei prodotti delle legende. Invece si limitò a disegnare nel 1842 una carta dell'isola a corredo della Guida della Sicilia di Giovanna Power, in cui evidenziava le produzioni vinicole siciliane e in particolare quelle di Marsala, dove operavano alcuni stabilimenti di proprietà britannica. Certo Marzolla non avrebbe immaginato che proprio la presenza delle industrie britanniche a Marsala avrebbe favorito lo sbarco di Garibaldi solo un paio d'anni dopo la sua morte. Infatti in quel fatidico 1860 le ditte britanniche e la banchina del porto godevano di una specie di extra-territorialità, e durante lo sbarco, al largo del litorale lilibeo una squadra di navi di linea della Royal Navy, al comando del contrammiraglio sir Rodney Mundy, era pronta ad intervenire per proteggere gli interessi britannici. In realtà per proteggere lo sbarco stesso sicché le navi napoletane inviate a contrastarlo esplosero solo poche cannonate, non potendo fare la guerra alla più potente marina militare del mondo.

Con l'Unità d'Italia ebbe fine la grande stagione della cartografia napoletana, almeno ufficialmente. In realtà i valenti tecnici del Reale Officio Topografico trasfusi nel nuovo organismo, Istituto Topografico Militare, poi IGM, contribuirono non poco a importanti realizzazioni come la prima carta topografica unitaria 1:50.000, che partì proprio dalla Sicilia.

Benedetto Marzolla e le sue carte, tuttavia, seguitarono ad essere conosciute, perché per alcuni anni dopo il 1861 restarono le uniche rappresentazioni attendibili, 
anzi le migliori, in attesa delle nuove carte. La fama del brindisino non si spense mai nella sua città di nascita, come importante gloria locale. Ancora nel 1909 il canonico Pasquale Camassa, erudito di storia brindisina, in un suo studio sui Brindisini illustri ricordava che Benedetto Marzolla fu "apprezzato da uomini sommi e remunerato da sovrani, tra cui Niccolò e Alessandro II di Russia” (Camassa 1909, 64).

Possiamo essere d'accordo perché Marzolla resta uno dei personaggi culturali di maggior spessore del Mezzogiorno preunitario, non solo per le produzioni cartografiche. Al Settimo Congresso degli Scienziati Italiani (1845), perorava una toponomastica all'avanguardia, e in scritti propositivi, di cui abbiamo dato conto in passato (Manzi 2007a) assieme ad altri ricercatori, evidenziava una concezione della geografia notevolmente avanzata, ricca di contenuti culturali come di pratica utilità.

\section{Conclusioni. "Marzolla Benedetto di Brindisi, geografo ed ufiziale del R. Ufficio topografico"}

Il termine "geografo" veniva spesso usato, tra Settecento e Ottocento, per qualificare il cartografo rilevatore, l'autore completo di carte, ma non il semplice esecutore. Giovanni Antonio Rizzi Zannoni, di fatto fondatore dell'ente cartografico napoletano, godeva della qualifica ufficiale di "regio geografo". Tuttavia nel caso del Marzolla la qualifica di geografo è meritata in senso più ampio, perché il personaggio è noto ai più appunto come autore di cartografia, e molto meno come geografo a tutto tondo, con ampie vedute mentali e tecniche parecchio avanti ai suoi tempi. Non un'eccezione negli ultimi decenni di vita dell'antico Regno, allorché diversi primati produttivi e tecnici mostravano una realtà diversa (almeno in una élite) da quella talora propagandata, piena di ignoranza, cattiva indole razziale, indolenza congenita, incapacità di sfruttare i "doni di natura" abbondanti in un mitico Sud baciato dalla fortuna ma incompreso dai suoi abitanti. Più o meno, fatte le debite proporzioni e considerando la maggiore rozzezza di linguaggio, le medesime accuse rivolte ai meridionali in varie occasioni e in tempi diversi. La "geografia" di Marzolla è universale, aperta al confronto, spaziosa nei suoi orizzonti concettuali e pluriregionali (Marzolla 1852).

Forse i geografi italiani attuali potrebbero trarne spunti beneauguranti per ricerche foriere di un'auspicata rinascita per un settore scientifico in crisi identitaria irrisolta.

Un'ulteriore dimostrazione di questo assunto può derivare da una scorsa alle teorie e applicazioni cartografiche d'avanguardia dei geografi e territorialisti francesi raccolti attorno alla rivista Mappe-monde e al gruppo scientifico Reclus capeggiato da Brunet e Ferras. L'avanguardia di alta classe geo-cartografica era ampiamente presente già un trentennio addietro, sicché le tesi mostrate e la maestria grafica adoperata sono valide ancor oggi, basta applicare la digitalizzazione senza confondere il mezzo con il fine, come invece specie in Italia si fa. D'altronde la validità degli esperimenti di Reclus viene riaffermata da un semplice paragone di spessore scientifico: nell'ambito della geografia italiana, salve alcune eccezioni, Mappe-monde, Reclus e parecchio altro prodotto in Francia viene quasi ignorato sia a livello didattico sia a livello di pubblicazioni scientifiche. Tra le eccezioni si possono ricordare Mario Cataudella e anche Margherita Azzari, specie per l'Atlante Geo-ambientale della Toscana (2006), due tra i maggiori esperti di cartografia tematica, in questa veste poco noti a buona parte dei geografi italiani, talora intenti a discutere su che cosa è o non è la geografia e se è abbastanza di sinistra (o di destra). Va ricordato anche Roberto Melis, direttore del Servizio cartografico del Touring Club Italiano, finché questo eccellente ramo del TCI è esistito. Melis, oltre che tecnico di alto livello, è stato anche un geografo tout court, nonché professore incaricato di corsi geocartografici in Università italiane, tra cui Pavia. Poiché la cartografia geografica poco si presta alle eterne diatribe inconcludenti, essa viene sempre più emarginata, nonostante l'impegno di alcuni settori di ricerca, come ad esempio l'AIC e il suo periodico e, per il ramo geostorico, il Centro Italiano di Studi Storico-Geografici (CISGE).

La carte, mode d'emploi è un manuale redatto nel 1987 da Roger Brunet per Fayard/Reclus, la lettura del quale fa capire perché la pianificazione territoriale in Francia è tanto diffusa, mentre in Italia è di fatto ignorata oppure applicata in maniera confusa, saltuaria e contraddittoria. Spinta solo da mode e contro-mode politiche di corto respiro. Cartografia bieco strumento del potere, buona per la guerra, mappe catastali e cabrei sette-ottocenteschi scambiati per produzioni "del popolo" perché spesso furono redatte a mano da agrimensori, studi notarili e cartografi locali, dimenticando che "il popolo" del tempo era in gran misura analfabeta e che quindi quei tecnici facevano comunque parte di un'élite, poca considerazione per i tesori del passato, come le opere di Benedetto Marzolla.

Anche l'attenzione recente per la Carta dei Prodotti alimentari si deve in gran parte all'equivoco di attualizzare realtà territoriali e produttive del passato pur continuando a seguire linee concettuali storico-scolastiche e legate all'idea del "progresso" tecnico. Marzolla viene comunque dal profondo Sud, quindi deve rappresentare 
un'eccezione in un mondo culturalmente "discutibile". Non si dice apertamente, eppure recenti e meno recenti lavori geografici o storico-geografici italiani pur di ampio respiro si arrestano, come ampiezza territoriale, all'Italia centrale, con qualche raro spunto su Napoli, ad esempio, Quaini (2009) e Gambi (1972), pur in ambiti scientifici di alta qualità.

La Carta alimentare di Marzolla pare, ad una prima occhiata, poco più che una divertente immagine di "disegnini” carini sparsi su una carta dell'Italia del Sud. Ma un esame più accurato, tenendo a mente i parametri di giudizio della cartografia di qualità (immediatezza, accuratezza, dati racchiusi in simboli chiari e comunicazione di concetti complessi in modo semplice) fa comprendere quanto avanzate fossero le concezioni del grande cartografo e geografo brindisino e non certo perché precorrerebbe Petrini, lo slow food a $\mathrm{km}$ zero o i marchi DOC. In pratica è quasi un precursore di Brunet e Ferras, cioè di quanto più originale e avanzato ci sia nella cartografia geografica degli ultimi 40 anni. La sola carta non basta: di nuovo si consiglia la lettura del Dizionario marzolliano e quella della breve biografia vergata dal fratello Raffaele, tra le righe velate dall'affetto fraterno. Forse soprattutto Silvia Siniscalchi, oltre ovviamente a Vladimiro Valerio, pare aver colto più a fondo la grandezza tecnico-scientifica del personaggio. Con una lontana premessa, breve ma significativa, quella del vecchio articolo locale di Domenico Novembre (1972). Articolo infatti ignorato dai geografi italiani per tanti anni, ma pure da altri notevoli esperti del ramo, sia importanti studiosi che collezionisti.

\section{Riferimenti bibliografici}

André, A. (1980). Lexpression graphique : cartes et diagrammes. Parigi, Masson.

Assessorato all'Agricoltura, Regione Campania (2006), Asprinio di Aversa. www.agricoltura.regione.campania.it/ Tipici/depliantvini/ ASPRINIO.pdf.

Atti della Settima Adunanza degli scienziati italiani tenuta in Napoli (1845). Napoli, Fibreno, 616-617.

Azzari, M. (2006). Atlante geoambientale della Toscana. Firenze, Regione Toscana. Novara, Istituto Geografico De Agostini.

Bianchini, L. (1839). Della storia delle finanze del Regno di Napoli. Napoli, Stamperia di F. Lao.

Bidischini, E., Musci, L. (1996). Guida agli archivi storici delle Camere di commercio italiane. Roma, Ministero per i Beni Culturali e Ambientali Ufficio Centrale per i beni archivistici (Strumenti, CXXVII).
Brancaccio, G. (1991). Geografia, cartografia e storia del Mezzogiorno. Napoli, Guida.

Brunet, R. (1987). La carte, mode d'emploi. Parigi, Fayard/ Reclus.

Brunet, R. (1997). Champs et contrechamps. Raisons de géographie. Parigi, Belin.

Buccaro, A. (1828). Pianta della città di Napoli e de' suoi contorni. Napoli, ROT.

Buttà, G. (1877). I Borboni di Napoli al cospetto di due secoli. Napoli, Tipografia del Giornale "La Discussione", Vol I., Vol. I, 21-51, Vol. III, 71-82.

Camassa, P. (1909). Brindisini illustri. Brindisi. Tipografia del Commercio.

Cantile, A. (2007). Sulla nascita della cartografia ufficiale italiana: gesuiti, scolopi, laici e militari, tra le esigenze della polemologia, le occorrenze dell'amministrazione e le necessità della scienza. In Cantile, A. (a cura di). La cartografia in Italia: nuovi metodi e strumenti dal Settecento ad oggi. Firenze, Istituto Geografico Militare, 31-57.

Carrabetta, A. (2003). Il saggio catastale di Marzolla e il paesaggio agrario e culturale di Castellammare di Stabia tra Ottocento e Novecento. Rivista Geografica Italiana, 110, 329-346.

Celentano, A. (2004). I vini d'Italia. Giudicati da papa Paolo III (Farnese) e dal suo bottigliere Sante Lancerio. Capri, La Conchiglia.

Ciasca, R. (1957). Le trasformazioni agrarie in Calabria dopo l'Unità. Atti del $1^{\circ}$ Congresso Storico Calabrese (Cosenza, 1954). Roma, Coll. Meridionale, 357-354.

Ciccolella, D. (2012). Hommes de guerre, hommes d'affaires. Filangieri, Nunziante e la politica doganale nel Regno delle Due Sicilie dopo il 1824. Storia economica, 15 (2), 403-436.

Collezione delle leggi e de' decreti reali del Regno delle Due Sicilie (1820). Napoli. Dalla Real Tipografia del Ministero di Stato della Cancelleria Generale. Anno 1820, Semestre I.

Conti, S. (2005). Alimentazione nell'Italia centro-meridionale nel secolo XIX. Geografia dellalimentazione. Atti Ottavo Seminario internazionale di geografia medica, a cura di Palagiano C. e De Santis G. Perugia. Rux, 91-99.

Conti, S. (2008). Lagroalimentare nel Regno delle Due Sicilie in una carta di Benedetto Marzolla. In Scritti in onore di Carmelo Formica, a cura di Castiello N., Napoli, Sezione Scienze Geografiche, Dipartimento ELPT, Università degli Studi di Napoli “Federico II", 249-258. 
Conti, S. (2014). La cartografia dell'Ufficio Topografico del Regno per lo studio e la salvaguardia di alcune aree della Provincia di Terra di Lavoro. Geostorie, 22 (1), 55-70.

Cundari, G. (2018). Il mondo: una bella prigione. Napoli, Scientifica.

De Dainville, F. (1964). Le langage des géographes. Parigi, Picard.

De Fabrizio, A. (1925). Benedetto Marzolla geografo e cartografo brindisino (1801-1858). Annuario 1923-24 $R$. Ginnasio "B. Marzolla". Brindisi.

De Matteo, L. (1982), Politica doganale e industrializzazione nel Mezzogiorno (1845-1849). Napoli, Pironti.

Farinelli, A. (2020). Le Dogane di Confine tra Stato Pontificio e Regno delle Due Sicilie. Kirke, Avezzano.

Gambi, L. (1954). Carlo Afàn de Rivera e l'Officio Topografico di Palermo. Archivio Storico Messinese, serie II, 5, 29-38.

Gambi, L. (1970). Cartografia storica. La storiografia italiana negli ultimi 20 anni. Atti I Congresso Nazionale Scienze Storiche. Milano, Marzorati, 1361-1383.

Gambi, L. (1972). I valori storici dei quadri ambientali. Storia d'Italia. Vol. I. I caratteri originali. Torino, Einaudi, 3-60.

Granata, L. (1830). Economia rustica per lo Regno di Napoli. Vol. I, Napoli, Tip. N. Pasca.

Graziani, A (1960). Il commercio estero del Regno delle due Sicilie dal 1832 al 1858. Archivio economico dell'unificazione italiana, serie I, 10, 89.

Guarducci, A. (2006). Mappe e poteri. Pubbliche istituzioni e cartografia nella Toscana moderna e contemporanea (secoli XVI-XIX). Firenze, All'Insegna del Giglio.

Guarducci, A., Rossi, M. (2018) (a cura di). Storia della cartografia e cartografia storica. Geotema, 58 [numero monografico].

Guerrieri, G. (1975). La Biblioteca Nazionale "Vittorio Emanuele III" di Napoli. Napoli, Ricciardi.

Landi, G. (1977). Istituzioni di diritto pubblico del Regno delle Due Sicilie (1815-1861). Napoli, Giuffrè, tomo I, 330-339.

Lentini, R. (2004). Sviluppo della viticoltura ed enologia nell'area del trapanese nel XVIII e nel XIX secolo. Studi garibaldini, 4 (3), 27-34.

Lepre, A. (1969). Sui rapporti tra Mezzogiorno ed Europa nel Risorgimento. Studi storici, 10 (3), 279-291.
Manzi, E. (1977). L'urbanizzazione del paesaggio agrario nel Mezzogiorno attraverso la cartografia e Catalogo dei documenti cartografici esposti. Atti del XXII Congresso Geografico Italiano (Salerno 18-22 aprile 1975), Vol. I, 167-226.

Manzi, E. (1987). Tavola II (Provincia di Basilicata di B. Marzolla, 1831); Tavola III (Carta dei prodotti alimentari delle Provincie Continentali del Regno delle Due Sicilie di B. Marzolla, 1856). In Il territorio per immagini. Atlante della Basilicata (a cura di Cataudella M.). Potenza, Regione Basilicata, Giunta Regionale. Napoli, Istituto Grafico Italiano.

Manzi, E. (2006). LUCC paesaggio e Mediterraneo. Quello che le carte e il paesaggio dicono e nascondono. In Manzi, E. (a cura di). LUCC paesaggio e Mediterraneo. Roma, Società Geografica Italiana (Memorie della Società Geografica Italiana, LXXXI), 5-60.

Manzi, E. (2007a). Benedetto Marzolla (1801-1858), la Commissione di Statistica generale del Ministero dell'Interno delle Due Sicilie e il Catasto. L'Universo, 87 (1), 116-135.

Manzi, E. (2007b). Carlo Afàn de Rivera (1779-1852). La cartografia come strumento di pianificazione e sviluppo del Mezzogiorno. Bollettino della Società Geografica Italiana, serie XII, 12, 299-313.

Manzi, E.(2009a). Cartografia e uso del suolo in Sicilia tra Settecento e Novecento. In Terra di Sicilia. Roma, Società Geografica Italiana, 109-150.

Manzi, E. (2009b). Giardini parchi paesaggi mediterranei. Roma, Società Geografica Italiana (Memorie della Società Geografica Italiana, LXXXVI), 7-122.

Manzi, E. (2018). Tra Roma antica e Napoli: l'enigma irrisolto della cartografia aragonese. L'Universo, 98 (1), 114-147.

Mappe-Monde, Trimestrale internazionale di cartografia edito dal Groupement d'Interet Publique RECLUS. Varie annate dal 1990.

Mariani, G. (1859). Almanacco etrusco Cronologico, Statistico, Mercantile. Firenze, Tipografia di F. Mariani.

Marzolla, B. (1852). Grande dizionario geografico storico del Regno delle Due Sicilie corredato di carte di moderna e antica geografia, corografia, topografia e di geologia e idrografia; non che di piante e disegni di vario genere di architettura, archeologia, paesaggi costumi ec. Che fa parte, ed a maggior chiarimento, dell'Atlante Statistico Universale. Napoli, Reale Tipografia Militare.

Marzolla, R. (1858). Biografia del Cavaliere Benedetto Marzolla scritta da suo fratello Raffaele. Napoli, Stabilimento Tipografico del R. Ministero dell'Interno. 
Mastriani, R. (1842)., Esposizione della legge del 19 giugno 1826, sulle dogane del Regno delle Due Sicilie. Napoli, Dalla Tipografia Flautina.

Mazzarelli, G. (1930). Gli atlanti geografici di Benedetto Marzolla geografo napoletano della metà del secolo XIX. Messina, Tipografia Saitta e Caminiti.

Mazzetti, E. (2020). Pesci cantati e pesci pittati. Napoli, Libreria Dante e Descartes, 49-67.

Mori, A. (1922). La cartografia ufficiale in Italia e l'Istituto Geografico Militare. Roma, Stabilimento Tipografico per l'Amministrazione della Guerra.

Nicali, A. (1997), Storia delle Dogane. Profili Storici della Politica Doganale Italiana. Suppl. A: Notiziario Fiscale, A. 97, N. 3. Ed. curata ed aggiornata da Giuseppe Favale. Presentazione di Gianni Marongiu. Roma, Ministero Delle Finanze, Dipartimento Delle Dogane E Delle Imposte Indirette.

Novembre, D. (1972). Lopera cartografica di Benedetto Marzolla. Brundisii Res, 4, 25-33.

Oliva, G. (1986). I corpi di finanza del Regno delle Due Sicilie. Roma, Centro tipografico Fiamme gialle del Comando generale della Guardia di Finanza.

Ostuni, N. (1992), Finanza ed economia nel regno delle due Sicilie. Napoli, Liguori.

Palsky, G. (2004). Francois de Dainville et l'histoire de la cartographie: orientations traditionelles et thématiques nouvelles. In Bousquet-Bressolier, C., Francois de Dainville: Pionnier de l'histoire de la cartographie et de léducation. Parigi, Publications de l'Ecole Nationale des cartes, 81-99.

Pignatelli, G. (2006). Napoli: tra il disfar delle mura e l'innalzamento del muro finanziere. Firenze, Alinea.

Quaini, M. (a cura di) (2009). I paesaggi italiani. Fra nostalgia e trasformazione. Rapporto annuale 2009. Roma, Società Geografica Italiana.

Reccia, G. (2013). Il controllo economico finanziario in Napoli e Casali. I finanzieri atellani. Sant'Arpino, Istituto di Studi Atellani.

Rescigno, M.R. (2007). Allorigine di una burocrazia moderna. Il personale del Ministero delle Finanze nel Mezzogiorno di primo Ottocento. Napoli, ClioPress.

Scirocco, A. (1986). Dalla seconda restaurazione alla fine del Regno. In Galasso, G., Romeo, R. (a cura di), Storia del Mezzogiorno, vol. IV. Roma, Edizioni del Sole.

Siniscalchi, S. (2019). La carta "alimentare" di Benedetto Marzolla (1856) fra prodotti tipici e risorse territoriali.
Bollettino della Associazione Italiana di cartografia, 167, 68-78.

Topa, M. (1990). Cosi finirono i Borbone di Napoli. Splendori e decadenza di unantica dinastia. Napoli, F.lli Fiorentino.

Touring Club Italiano (1931). Guida gastronomica d'Italia. Milano.

Touring Club Italiano (1940). Atlante fisico-economico d'Italia, diretto da Dainelli G.

Touring Club Italiano, Consiglio Nazionale delle Ricerche. (1992). Atlante Tematico d'Italia. Milano. Tavole 68, $69,71,73$.

Valerio, V. (1980). Atlanti napoletani del diciannovesimo secolo (1806-1860). Napoli, Luigi Regina.

Valerio, V. (1985). L'Italia nei manoscritti dell'Officina Topografica. Napoli, Istituto Italiano per gli Studi Filosofici.

Valerio, V. (1993). Società uomini e istituzioni cartografiche nel Mezzogiorno d'Italia. Firenze, Istituto Geografico Militare. 1-752.

Valerio, V. (1998). Benedetto Marzolla nell'Officio Topografico di Napoli. Benedetto Marzolla disegnatore e cartografo brindisino. Atti del Convegno "Settimana della cartografia". Brindisi, Liceo classico Benedetto Marzolla, 21-32.

Valerio, V. (2002). Costruttori di immagini. Disegnatori, incisori e litografi nell'Officio Topografico di Napoli. Napoli, Paparo.

Valerio, V. (2008). Benedetto Marzolla. Brindisino, Geografo e Cartografo dell'800 Europeo. Manduria, Barbieri Selvaggi. 